\title{
Separating homeologs by phasing in the tetraploid wheat transcriptome
}

\author{
Ksenia V Krasileva', Vince Buffalo', Paul Bailey², Stephen Pearce', Sarah Ayling², Facundo Tabbita', Marcelo Soria ${ }^{1,3}$, \\ Shichen Wang ${ }^{4}$, IWGS Consortium ${ }^{5}$, Eduard Akhunov ${ }^{4}$, Cristobal Uauy ${ }^{6}$ and Jorge Dubcovsky ${ }^{1,7^{*}}$
}

\begin{abstract}
Background: The high level of identity among duplicated homoeologous genomes in tetraploid pasta wheat presents substantial challenges for de novo transcriptome assembly. To solve this problem, we develop a specialized bioinformatics workflow that optimizes transcriptome assembly and separation of merged homoeologs. To evaluate our strategy, we sequence and assemble the transcriptome of one of the diploid ancestors of pasta wheat, and compare both assemblies with a benchmark set of 13,472 full-length, non-redundant bread wheat cDNAs.

Results: A total of 489 million 100 bp paired-end reads from tetraploid wheat assemble in 140,118 contigs, including $96 \%$ of the benchmark cDNAs. We used a comparative genomics approach to annotate 66,633 open reading frames. The multiple k-mer assembly strategy increases the proportion of cDNAs assembled full-length in a single contig by $22 \%$ relative to the best single $\mathrm{k}$-mer size. Homoeologs are separated using a post-assembly pipeline that includes polymorphism identification, phasing of SNPs, read sorting, and re-assembly of phased reads. Using a reference set of genes, we determine that $98.7 \%$ of SNPs analyzed are correctly separated by phasing.

Conclusions: Our study shows that de novo transcriptome assembly of tetraploid wheat benefit from multiple kmer assembly strategies more than diploid wheat. Our results also demonstrate that phasing approaches originally designed for heterozygous diploid organisms can be used to separate the close homoeologous genomes of tetraploid wheat. The predicted tetraploid wheat proteome and gene models provide a valuable tool for the wheat research community and for those interested in comparative genomic studies.
\end{abstract}

Keywords: Transcriptome assembly, multiple k-mer assembly, wheat, polyploid, Triticum urartu, Triticum turgidum, pseudogenes, phasing, gene prediction

\section{Background}

Whole genome duplication events, or polyploidization, have occurred repeatedly throughout the evolutionary history of flowering plants[1,2]. Many currently cultivated species are recent polyploids, formed through either inter-specific hybridization (allopolyploids, such as wheat, oats, canola, peanut, and cotton)or intra-specific hybridization (autopolyploids, such as apple, strawberry, watermelon, and alfalfa)[2]. In addition,homoeologs in older polyploid species, such as maize (11-15 million years since polyploidization)[3] have had a longer time to diverge through deletions, loss of function, neo-functionalization, and sub-functionalization processes (usually

\footnotetext{
* Correspondence: jdubcovsky@ucdavis.edu

'Dept. Plant Sciences, University of California, Davis, CA 9561, USA

Full list of author information is available at the end of the article
}

referred to as diploidization). These processes confer polyploid species an increased evolutionary plasticity, whichpromotes speciation and adaptation to new environmentsand contributes to the huge success of polyploidy in plant evolution[2,4].When diploidization processes continue over long periods of time, they lead to the formation of paleo-polyploid species (for example, rice), which are difficult to differentiate from true diploid species. However, genomic studies haveprovided convincing evidence of ancient whole genome duplication events in the early monocot and dicot lineages suggesting that polyploidy was part of the evolution of most current angiosperms [5,6].

Wheat (Triticum spp.) was domesticated at the dawn of agriculture approximately 10,000 years ago and has since been adapted to grow in a broad range of climates
Ciomed Central

() 2013 Krasileva et al.; licensee BioMed Central Ltd. This is an open access article distributed under the terms of the Creative Commons Attribution License (http://creativecommons.org/licenses/by/2.0), which permits unrestricted use, distribution, and reproduction in any medium, provided the original work is properly cited. 
throughout the world [4]. Most cultivated wheat varieties belong to two species; tetraploid Triticum turgidum L. (durum or pasta wheat, genomes $\mathrm{AABB}$ ) and hexaploid T. aestivum L. (common wheat, genomes AABBDD). The tetraploid wheat genome originated from an inter-specific hybridization event occurring less than 0.5 million years ago, which combined the AA genome of T. urartu Tumanian ex Gandilyan and the $\mathrm{BB}$ genome of an unknown grass species related to Aegilops speltoides Tausch[7-9].Common wheat, T. aestivum, evolved from a second round of inter-specific hybridization and genome duplication that occurred shortly after domestication and combinedthe tetraploid AABB genomes of cultivated T. turgidum and the DD genome of the wild grass Aegilops tauschii (Coss.) Schmalh[4].

The diploid progenitors of polyploid wheat species diverged from a common ancestor only 2.5-4.5 million years ago[10], which is reflected in a high average identity (approximately 97\%) among coding regions of different wheat homoeologs. However, this average varies greatly among gene classes that are subject to different evolutionary pressures [11]. For example, conversion events (unequal crossing-over between tandemly-duplicated paralogs) and diversifying selection processes are known to accelerate the divergence rate between members of the disease resistance gene family [12-14].

Wheat intergenic regions diverge even faster than rapidly evolving gene families due to high levels of methylation and increased rates of insertions and deletions, which are associated with the abundance of repetitive elements in these regions [15]. These rapid changes in the intergenic regions can affect neighboring genes and result in rapid rates of gene insertion, deletion, and transposition [16].The potentially negative effects associated with gene deletions are buffered by polyploidy [17-20].Transposition of genes and gene fragments by adjacent retroelements results in higher proliferation of pseudogenes in the large polyploid Triticeae genomes compared to other grass species with smaller genomes [19,21].In addition, increased divergence of alternative splicing variants between the diploid progenitors further diversifieshomoeologs'gene structure (and potentially their function) in the polyploid wheat species[21]. The dynamic nature of these large genomes needs to be considered in the development of strategies to characterize the wheat gene complement.

In species with large genomes, de novo transcriptome assemblies are an effective strategy to access the gene spacewhile avoiding the highly repetitive intergenic regions. In wheat, for example, the transcribed genecoding regionsrepresent only $1 \%$ to $2 \%$ percent of the totalgenome[22]. Rapid growth in throughput, quality, and accessibility of next-generation sequencing technologies, together with improvements in de novo transcriptome assembly algorithms have fostered a multitude of transcriptome sequencing projects. With increased access to next generation sequencing, many plant de novo transcriptome assemblies have been published and several different assembly algorithms have been proposed[23-25]. However, the challenges specific to de novo transcriptome assembly of a young polyploid speciessuch as tetraploid wheat are just starting to be addressed[26,27]. Particularly important is the correct separation of close homoeologs, since there are known examples of different homoeologs contributing differently to important agronomic traits (for example, wheat VRN1 homoeologs[28]). Correct separation of homoeologs is also important for breeding applications, marker development, and downstream genomics analyses.

Three recent studiesof hexaploid wheat transcriptomes $[27,29,30]$ highlight the difficulties of assembling closely related homoeologs in a polyploid species. Schreiber et al. (2012) observed that most homoeologs were collapsed into chimeric contigs when hexaploid wheat transcriptomes were assembled using either Velvet/Oases (60\% to $80 \%$ chimeric sequences) or Trinity (50\% chimeric sequences). A computationally-intensive two-stage assembly using the MIRA assembler helped to reduce the number of chimeric homoeolog sequences to $18 \%$, thus partially solving the polyploid problem at the assembly step [27].An alternative strategy was used by The International Wheat Genome Sequencing Consortium (IWGSC): genome-specific contigs of hexaploid wheatwere generated by sorting individual chromosome arms usingflow cytometry and sequencing and assembling each of them separately[21,31,32].

In this paper, we present abioinformatics pipeline that addresses the challenges of de novo transcriptome assembly of the closely related genomes of tetraploid wheat. Using this pipeline, weassembled, annotated and analyzedthe transcriptome of T. turgidum cv. Kronos and of its closest diploid relative $T$. urartu. This diploid wheat transcriptome together with a reference dataset of 13,472 fulllengthwheat cDNAs were used to evaluate the effect of different parameters on the quality of the tetraploid wheat assembly.We developed post-assembly processing strategies and software that allowed us to generate homoeologspecific sub-assemblies. Finally, we used comparative genomics approaches to annotate open reading frames and predicted proteins, predict pseudogenes and artificially fused transcripts, and generate gene models to increase the value of this resource.

\section{Results and discussion}

\section{Sequencing and evaluation of experimental and digital} normalization

In total, we sequenced 248.5 million and 488.9 million paired-endIllumina reads (100 bp each) for $T$. urartu and T. turgidum cv. Kronos, respectively (Additional 
File 2, Table S1). The raw reads were submitted to the Short Read Archive (SRA) and linked to their respective NCBI BioProjects PRJNA191053(T. urartu) and PRJNA191054(T. turgidum). After trimming Illumina adapter sequences with Scythe and poor quality bases with Sickle (see Materials andmethods) the average read length was94 bp for $T$. urartu and 96 bp for T. turgidum. The number of reads obtained from individual RNA-seq libraries varied from 20.3 to 137.1 million reads and is summarized in Additional file 2, Table S1.

\section{Double-stranded DNA nuclease (DSN) normalization}

Results from DSN are described in Figure S1 (Additional file 3). First, we evaluated the fold change in abundance of four marker genes by quantitative RT-PCR (Additional file 3, Figure S1A). Rubisco, one of the most highly expressed genes, showed an 11- to 13-fold decrease in transcript levels after normalization, whereas transcripts of a low abundance NBS-LRR geneshowed a slight increase after normalization (Additional file 3, Figure $\mathrm{S} 1 \mathrm{~A})$. We then evaluated the relative abundance of Illumina reads mapped to a reference set of full-length wheat cDNA transcripts and additional high abundance geneswith and without normalization (Additional file 3, Figure S1B,C). Our results showed that DSN normalization resulted in an enrichment of the low abundance transcripts and a reduction of the most abundant transcripts relative to the control without DSN normalization. There were a substantial number oftranscripts detected only after normalization (new points to the left of the red reference line in Figure S1C), which indicates that our DSN normalization contributed to a more comprehensive transcriptome assembly.

\section{Digital normalization}

In addition to the experimental DSN normalization and prior to assembly, we performed a digital normalization of the reads using the khmerprogram [33] (see Materials and methods). This normalization is designed to reduce redundancy in the RNA-seq data and accelerate assembly. We tested the effect of digital normalization on assembly quality using a previously published RNA-seq library of T. turgidum cv. Langdon [34]. The 28 million reads present in this library were reduced to 9 million reads after digital normalization. Both sets of reads were assembled using our multiple k-mer size assembly pipeline (see next section) and the resulting contigs were aligned to the 13,472 full-length wheat cDNA benchmark set [35] using BLASTN (E-value $1 \mathrm{e}^{-20},>90 \%$ identity). Additional file 4 , Figure S2 shows thatboth datasets have identical distributions of the number of reference genes assembled at different levels of coverage (correlation between distributions $R=0.99989$ ). This result confirmed that digital normalization had no significant negative effects on the quality of assembliesgenerated by our multiple k-mer length assembly pipeline. Digital normalization reduced the number of paired-end reads five-fold (Table 1), thus greatly reducingthe time and resources required for the multiple k-merassemblies.

\section{Distribution of percent identity and SNP distances between $\mathrm{A}$ and $\mathrm{B}$ homoeologs}

Several of the programs used in our assembly pipeline require input parameters that are dependent on the level of divergence between the homoeologousgenomes and/ oron the average distance between single nucleotide polymorphisms (SNPs). To estimate these two parameters we analyzed the coding sequences of 52 genes (26 A/B genome homoeolog pairs, average size 1,199 bp, Supplemental dataset $1[36]$ ), which were previously sequenced and annotated in our laboratories. DNA sequence identity (excluding gaps) between A and B coding regions showed a normal distribution (Shapiro-Wilk test $P=0.40$ ) with a mean of $97.26 \%$ and a standard deviation of $1.20 \%$ (Figure 1A). Based on this result,we estimated that a minimum threshold of $94 \%$ identity ( $\leq 12$ SNPs per $100 \mathrm{bp}$ paired-end fragments) would include approximately $99 \%$ of all identity values between true homoeologs and allow roughly $99 \%$ of the paired-end reads to map to both homoeologs. With a minimum threshold of $95 \%$ identity ( $\leq 10$ SNPs per $100 \mathrm{bp}$ paired-end fragments) the previous proportions were reduced to $95 \%$ of the homoeologs and mapped reads.

Poisson processes for SNPs imply exponential distributions of inter-SNP distances and hence long tails [37]. The frequency of inter-SNP distances found in this study between wheat homoeolog coding regions also decreased exponentially with inter-SNP distance (Figure 1B). For this set of 52 genes, the mean distance between adjacent SNPs was 37.8 bp (standard deviation of 47.1 $\mathrm{bp})$, which is close to the average distance of $32.9 \mathrm{bp}$ estimated from the $97.26 \%$ percent identity and the $1,199 \mathrm{bp}$ average lengthof the 26 manually-curated homoeolog pairs used in our dataset (Figure 1B).

This level of polymorphism, the variable distances between adjacent SNPs, and the need to separate close homoeologspose challenges to most transcriptome assemblers, which were designed and tested for lower levels of intraspecific heterozygosity and were not required to separate close haplotypes. To address this problem we applied several post-assembly processing tools that integrateavailable and novel software to generate homoeolog-specific sub-assemblies. The overall assembly strategy is described in Figure 2A, the annotation procedures in Figure 2B, and the specific steps to separate the collapsed homoeologs into homoeolog-specific sequences are illustrated in Figure 2C. A detailed description of each of the different steps is included below. 
Table 1 The $T$. urartu and $T$. turgidum final assembly statistics

\begin{tabular}{lll}
\hline & T.urartu & T.turgidum \\
\hline 100-bp paired-end reads $(n)$ & 248.5 million & 488.9 million \\
Reads after digital normalization $^{\text {a }}(n)$ & 47.3 million & 110.7 million \\
Contigs $(n)$ & 86,247 & 140,118 \\
Mean contig size (bp) & $1,417 \mathrm{bp}$ & $1,299 \mathrm{bp}$ \\
Min contig size (bp) & $212 \mathrm{bp}$ & $298 \mathrm{bp}$ \\
Max contig size (bp) & $17,959 \mathrm{bp}$ & $26,226 \mathrm{bp}$ \\
GC content (\%) & $49 \%$ & $49 \%$ \\
Total transcriptome size (Mb) & $122 \mathrm{Mb}$ & $181 \mathrm{Mb}$ \\
Reads mapping to the assembly (\% of total reads) & $82.2 \%$ & $81.5 \%$ \\
Reads mapped in proper pairs (\% of total reads) & $73.0 \%$ & $71.5 \%$ \\
Unique alignments (\% of total mapped) & $52.8 \%$ & $76.7 \%$ \\
Benchmark genes ${ }^{\mathrm{b}}$ assembled $>50 \%$ length in a single contig & $12,693(94 \%)$ & $12,961(96 \%)$ \\
Benchmark genes ${ }^{\mathrm{b}}$ assembled $>90 \%$ length in a single contig & $10,727(80 \%)$ & 10,197 (76\%) \\
\hline
\end{tabular}

${ }^{a}$ Elimination of Homo sapiens,Escherichia coli, wheat mitochondrial, rRNA, and chloroplast sequences resulted in the elimination of $0.5 \%$ of the digitally normalized reads in $T$. urartu and $0.6 \%$ in $T$. turgidum.

b 13,472 full-length cDNAs from the RIKEN Plant Science Center Japan [35].

\section{Effect of different k-mersizeson the assembly of diploid and tetraploid wheat transcriptomes}

For initial reconstruction of the wheat transcriptome we useda de Bruijn graph de novo assembly algorithm implemented in CLC Genomics v5.5. Since the word size (or $\mathrm{k}$-mer size) is one of the key parameters in constructing de Bruijn graphs, we evaluated the effect of 10 different k-mer sizes(ranging from 21 to 63, the maximum permitted in CLC) on the assembly of tetraploid and diploid wheat
transcriptomes.At each k-mer size, we assessed basic assembly metrics, includingthe total number of contigs, average contig size and the proportion of reads assembled. In addition to these basic measures, we estimated completeness of our assemblyby assessing the proportion of 13,472 benchmark cDNA sequences[35] assembled at full length in a single contig (Figure 3A-E, Additional file 2, Table S2).

While the resulting number of contigs and average contig size differed very little across the range of $\mathrm{k}$-mer

\section{A \\ Distribution of \% identity among experimentally validated $\mathrm{A} / \mathrm{B}$ genes}

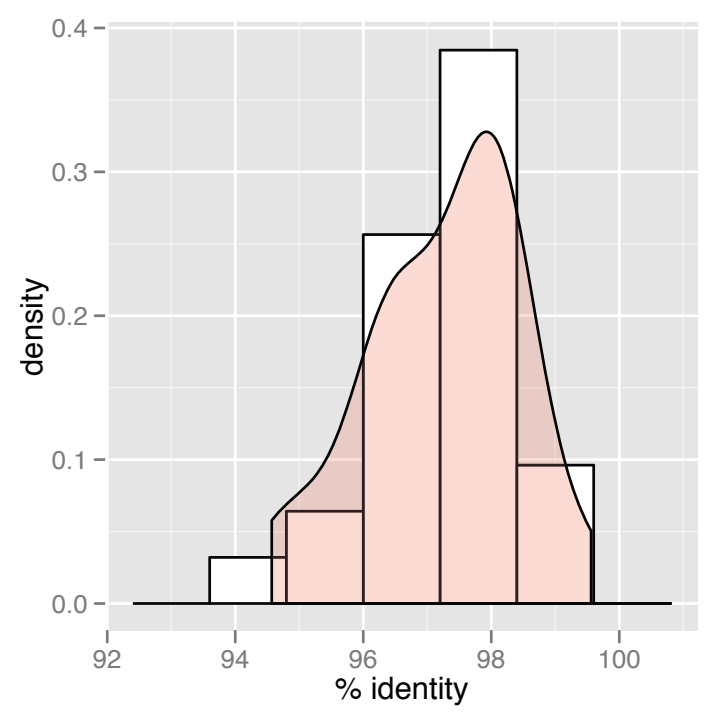

B

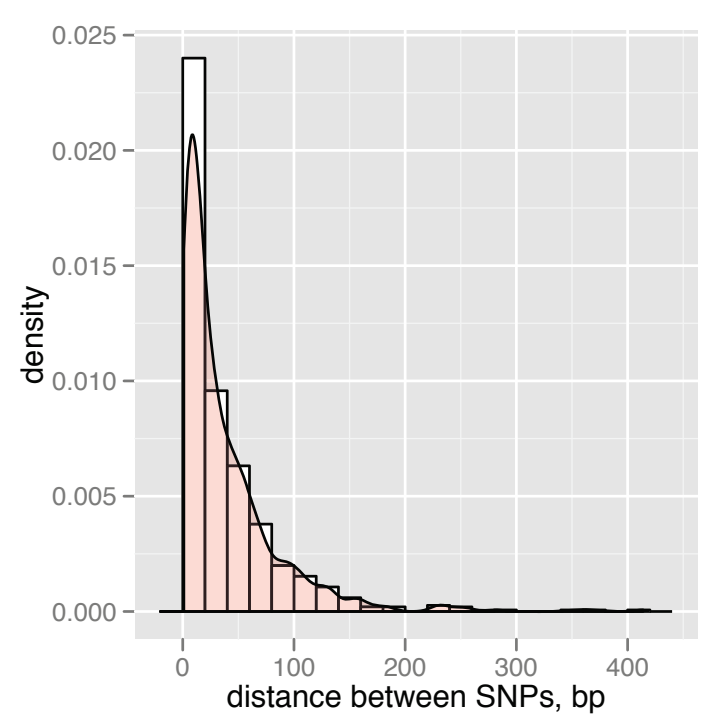

Figure 1 Divergence of A and B transcripts. (A) Distribution of percent identity between A/B homoeologous genes in a set of 26 experimentally validated genes (52 homoeologs). Mean $=97.3 \%$; SD $=1.20 \%$. (B) Distribution of distances between 707 single nucleotide polymorphisms (SNPs) between homoeologs in tetraploid wheat coding regions. Mean = 37.8 bp; SD = $47.1 \mathrm{bp}$; Median = $27 \mathrm{bp}$. 


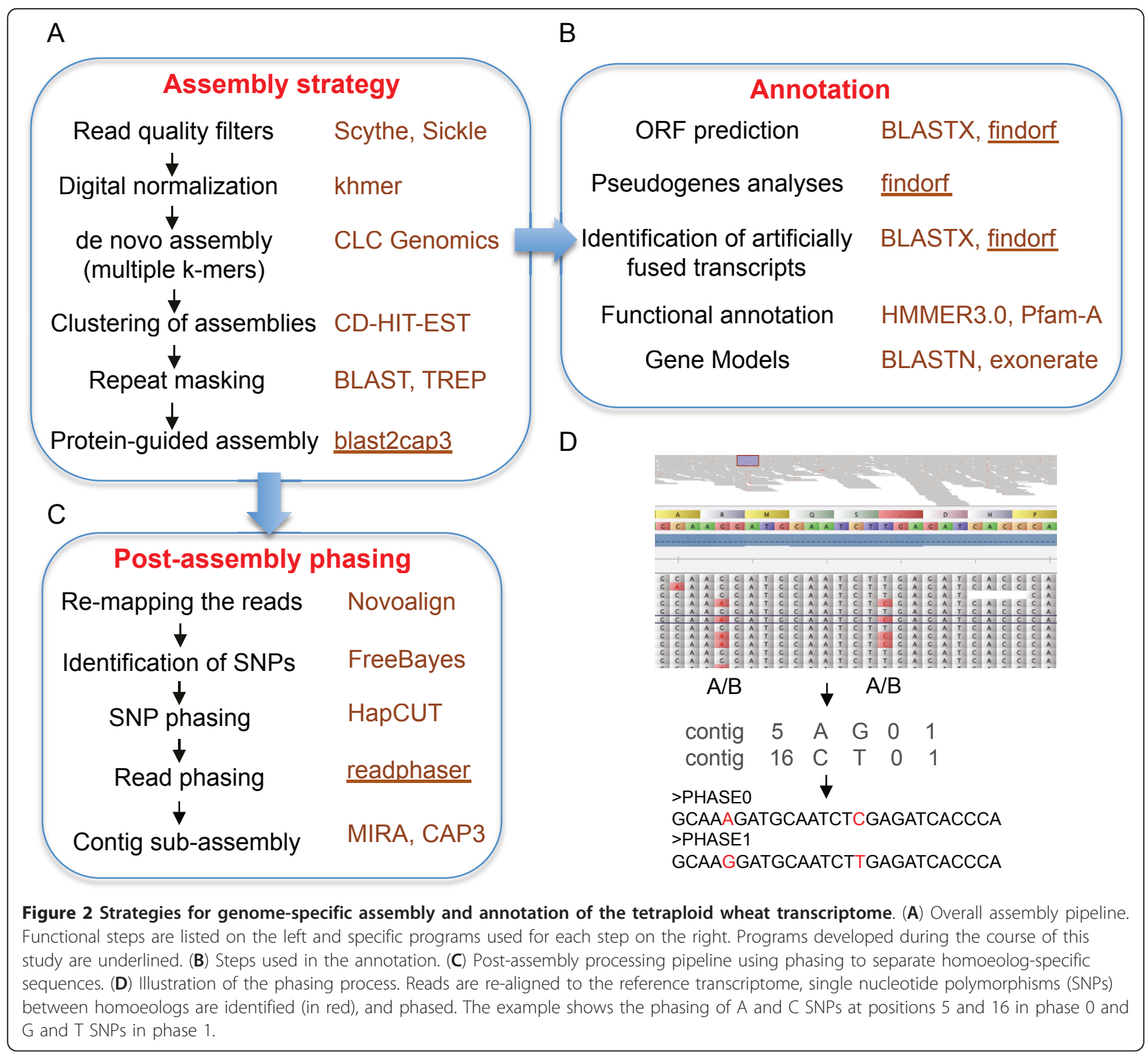

values (Figure 3A, B, Additional file 2 Table S2), all other metrics indicated an improvement in assembly quality with increased k-mersize up to k-mer 61 (Figures 3C, D, and 3E, Additional file 2 Table S2). For both T. urartu and T. turgidum, assembly completeness increased with k-mer length; at k-mer 63, 74\% and 70\% of all reads utilized in the assembly compared to only $56 \%$ and $52 \%$ at k-mer 21, respectively (Figure 3C, Additional file 2 Table S2). The percent of reads mapping in proper pairs, an indicator of assembly continuity also improved with increasing k-mer size, but the gain was more modest, ranging from $61 \%$ at k-mer 21 to $63 \%$ to 64\% at k-mer 63 (Figure 3D, Additional file 2 Table S2).

Figure $3 \mathrm{E}$ shows that a larger proportion of the 13,472benchmark cDNA sequences [35] are assembled at full length ( $>90 \%$ coverage) at larger k-mersizes. This metric showed clear differences between the diploid and the tetraploid assemblies for all k-mersizes, with the T. turgidum assemblies showing a lower proportion of fully assembled genes than the T. urartu assemblies. In T. turgidum, only $46 \%$ of benchmark genes were assembled at fulllength in a single contig at k-mer 63, while in T. urartu, this number was close to $60 \%$ (Figure 3E, F, Additional file 2 Table S2). This result suggeststhat de novo transcriptomeassemblies can be more fractionated in polyploid species with recently duplicated genomes than in their donor diploid species.

Triticum turgidum contigs that are separated correctly into A and B homoeologs should show a bimodal distribution of percent identities when compared with 


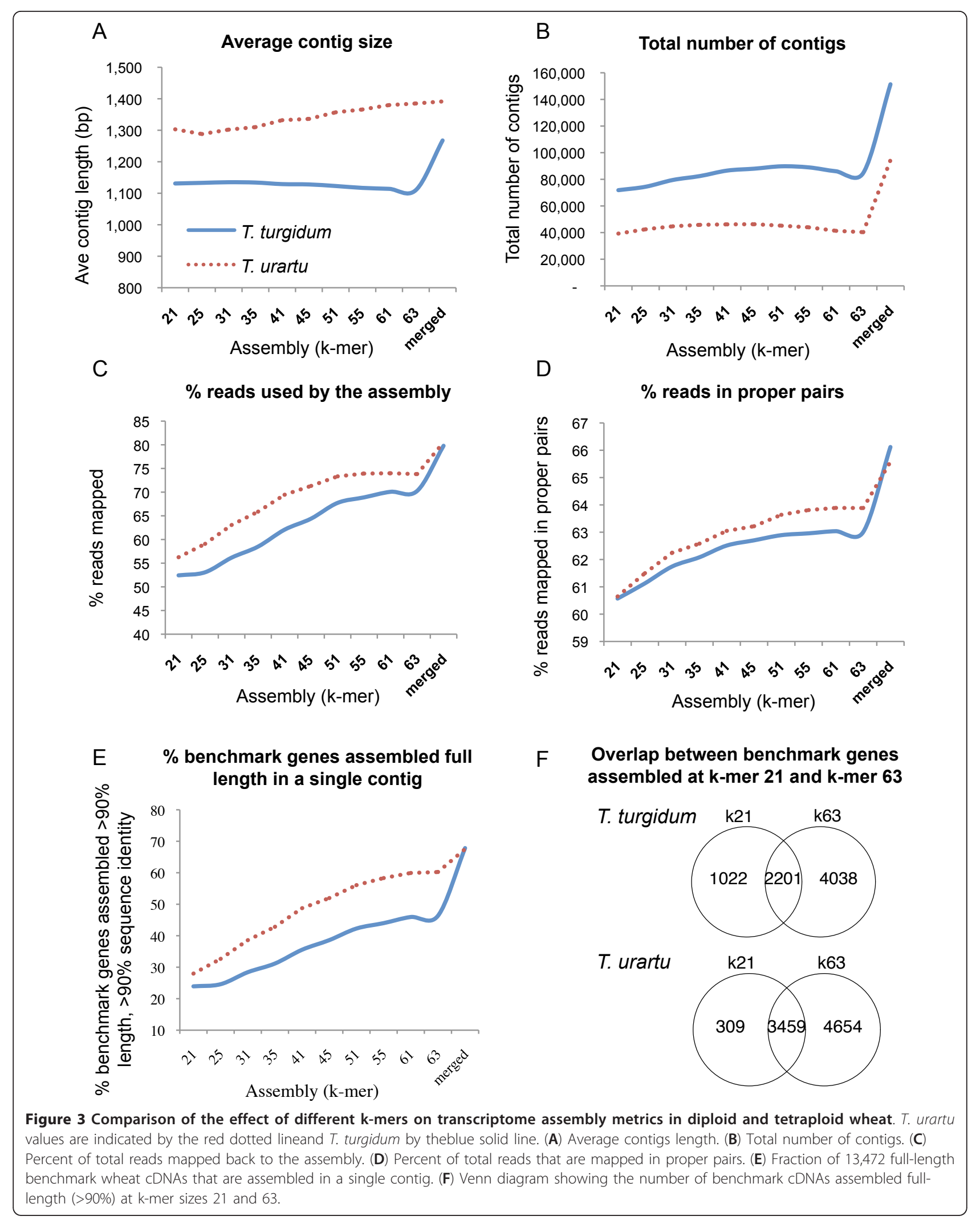


T. urartu. In Figure 4, we plotted the distribution of percent identities between the best BLAST hits between T. urartu and T. turgidum contigs, colored according to the specific k-mer assembly that contributed that contig. All k-mer sizes show a sharp peak at $99 \%$ identity that corresponds to the tetraploid A genome contigs aligned with the diploid A genome progenitor, but only the larger k-mersizes show a second peak around $96 \%$ identity (Figure 4). We interpret this result as evidence of a better separation of $\mathrm{A}$ and $\mathrm{B}$ genome contigs derived from the larger $\mathrm{k}$-mersizes. Chimeric $\mathrm{A} / \mathrm{B}$ assemblies are more abundant at lower k-mersizesand their intermediate percent identity values 'fill' the valley between the $\mathrm{A} / \mathrm{A}$ and $\mathrm{A} / \mathrm{B}$ peaks resulting in curves with a single peak (Figure 4). For all k-mersizes, identity values $<94 \%$ include the most divergent $1 \%$ of the homoeologs, but most likely also include many paralogous alignments.

\section{Advantages and disadvantages of merged multiple k-mer assemblies}

Since different k-mer sizes lead to full-length assemblies ofdifferent sets of genes (Figure 1F), we combined the contigs from the 10 different $\mathrm{k}$-mer assemblies, and eliminated redundancy using the CD-HIT program (see Materials and methods), which was recently shown to produce more inclusive sets of transcripts compared with Oases and V-MATCH [38].

The advantages of this approach were evident in the improvement of several assembly metrics in the CD-HIT merged $\mathrm{k}$-mer assemblies relative to the single $\mathrm{k}$-mer assemblies. The CD-HIT merged k-mer datasets showed a higher fraction of reads mapping back to the assembly (Figure 3C), a higher fraction of reads mapped in proper pairs (Figure 3D), and an increase in the proportion of contigs including complete benchmark transcripts (Figure 3E) in both T. turgidum and T. urartu. However, gains from the merged dataset relative to the best individual k-mer size were greater in $T$. turgidum than in $T$. urartu. This difference was particularly clear for the last metric, where the percent of cDNAs assembled full-length in a single contig rose from $60 \%$ to $68 \%$ for T. urartu and from $46 \%$ to $68 \%$ for T. turgidum (Figure 3E). It is interesting to point out that, based on our different metrics, the quality of our diploid and tetraploid transcriptomes are similar only in the merged $\mathrm{k}$-mer assemblies. This result suggests that by merging assemblies from a wide range of $\mathrm{k}$-mersizes it is possible to achieve similar quality for both diploid and tetraploid de novo transcriptome assemblies. The importance of using a wide range of k-merlengthsin the assembly of transcriptomes from polyploid species is further illustrated

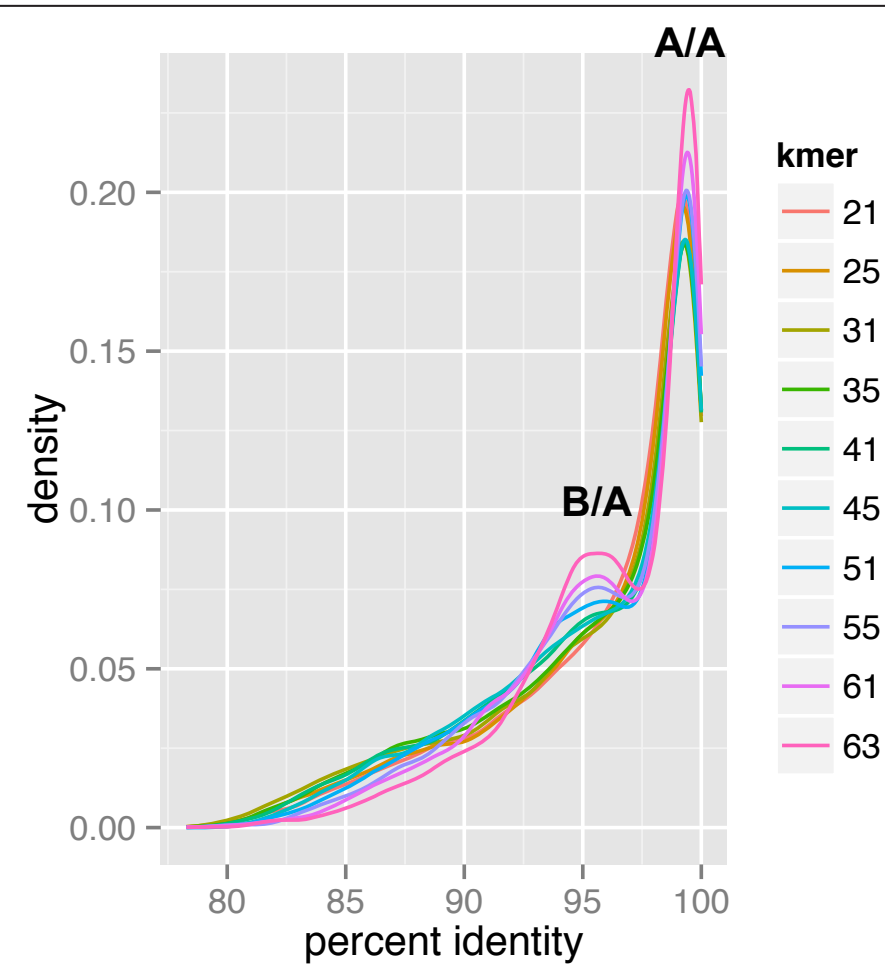

Figure 4 Distribution of percent identities between $T$. urartu and $T$. turgidum merged assemblies. The graph represents the distribution of percent identity between T. turgidum and T. urartu merged assemblies as calculated by BLASTN(E-value cutoff $1 \mathrm{e}^{-20}$ ). Densities are colored by the k-mer which contributed each contig to the merged assembly. 
in Figure 3F, which shows that the proportion of genes assembled at full length at k-mer 21 but not at k-mer 63 was larger in T. turgidum (14\%) than in T. urartu (4\%, Figure 3F).

One disadvantage of using a multiple k-mer approach is the high redundancy generated by duplicated genes and different $\mathrm{A} / \mathrm{B}$ chimeric forms of the same gene assembled at different k-mersizes. Fortunately, a large proportion of this redundancy can be eliminated using CD-HIT with the appropriate percent identity threshold. By adjusting this parameter to $95 \%$ in T. turgidum and $99 \%$ in $T$. urartu we reduced the initial number of contigs 4.6-fold in T. urartu and 5.5-fold in T. turgidum (Additional file 2, Table S2). A 95\%identity threshold was selected for tetraploid wheat to merge most (approximately 95\%) duplicated chimeric contigs (Figure 1A). This thresholdalso eliminated one member of fully-overlapping homoeolog-pairs even if they were not A/B chimeras. Many of the eliminated homoeologs were recoveredat a later stage during the phasing and reassembly of phased reads (Figure 2C). As mapping quality decreases significantly when reads map to multiple locations, the elimination of one of the members of close homoeolog pairs has the additional benefit of improving read mapping quality which is important for SNP calling and phasing in the next steps of our pipeline.

A limitation of the CD-HIT program is that it does not merge partially overlapping contigs, so additional steps were needed to combine overlapping contigs from different k-mer assemblies. To reconstructtranscripts split between partially overlapping contigs we implemented blast2cap3[39], a protein-guided assembly approach,to reconstruct partially overlapping contigs assembled at different k-mersizes (see Materials and methods, Additional file 5). Briefly, blast2cap3first clusters contigs based on similarity to a common protein and then passes each cluster to the overlap-based assembly program CAP3[40]. By operating on small subsets of contigs that have been pre-filtered using biologically-relevant information, blast2cap3 generates less artificially fused sequences as compared to assembling the entire dataset with CAP3. For this study we used seven plant protein databases (six grass species and Arabidopsis, see Additional file 2, Table S3) and a high stringency criteria (>99\% identity for $>100 \mathrm{bp}$, to reduce the generation of chimeric A/B clones). To further lower the risk of merging incorrect contigs based on common repetitive elements we masked all sequences using the Triticeae Repeat Sequence Database (TREP) (BLASTN and BLASTX, E-value cutoff $1 \mathrm{e}^{-10}$ ) before running blast2cap3. The implementation of blast2cap3 reduced the total number of contigs by $8 \%$ to $9 \%$, reducing assembly redundancy and/or fractionation.
After these merges,the final transcriptomes included 86,247 contigs for $T$. urartu (average 1,417 bp, Supplemental dataset 2[36]) and 140,118 contigs for T. turgidum (average 1,299 bp, Supplemental dataset 3[36]) (Table 1). The T. turgidum transcriptome included $96 \%$ of the 13,472 benchmark full-length cDNA sequences [35] with a coverage $>50 \%$ in single contigs and $80 \%$ with a coverage $>90 \%$ (compared to 68\% before blast2cap3). The T. urartu transcriptome showed similar parameters $(94 \%$ with coverage $>50 \%$ and $76 \%$ with coverage $>90 \%$, Table 1 ). These results suggest that our transcriptomes include a large proportion of all wheat genes. Final T. urartu and T. turgidum assemblies were filtered according to the guidelines of Transcriptome Shotgun Assembly (TSA) and deposited under TSA accessions GAKL00000000 and GAKM00000000, respectively.

After the assemblies were completed, the bioinformatics pipeline branched in two directions: one focused on the annotation of the contigs (Figure 2B) and the other aimed at separating sequences from the $\mathrm{A}$ and $\mathrm{B}$ genomes by phasing (Figure $2 \mathrm{C}$ ). These post-assembly processes are described in detail below.

\section{Open reading frame prediction and functional annotation of wheat transcriptomes}

The ORF prediction process was based on a comparative genomics approach implemented in the findorf program (Additional file 6) [41]. This approach relies on BLASTX alignments between transcripts and proteomes from other plant species (Additional file 2, Table S3) and Hidden Markov Model (HMM)-based Pfam domain predictions (see Materials and methods for specific parameters). In total, we predicted 76,570 ORFs for T. turgidum (and 43,014 for T. urartu, Table 2).Functional annotation of the predicted proteins using HMM-based searches against Pfam[42] (see Materials and methods) showed that the three most prominent domains in our wheat transcriptomeswereprotein kinase (Pkinase), leucine-rich repeat (LRR), and nucleotide-binding site (NBS) domains - signature domains of receptor-like kinases and plant disease resistance genes.

Roughly $30 \%$ of the contigs $(26,070$ for $T$. urartu and 42,999for T.turgidum) did not show significant similarity to any plant protein by BLASTX (E-value $1 \mathrm{e}^{-3}$ ), nor to any Pfam domain (E-value $1 \mathrm{e}^{-3}$ ) (Table 2). These contigs are likely to include:(1) wheat-specific genes and rapidly evolving gene families;(2) expressed pseudogenes that have accumulated too many mutations; (3) non-coding transcribed sequences; (4) pieces of 5' and 3' UTRs; and (5) general assembly artifacts. Although at this point it is hard to differentiate between these possibilities, it is interesting to note that many well-studied transcriptomes, such as mouse and human, contain a substantial number of long 
Table 2 Open reading frame prediction ${ }^{\mathrm{a}}$

\begin{tabular}{|c|c|c|}
\hline & T. turgidum & T. urartu \\
\hline Contigs $(n)$ & 140,118 & 86,247 \\
\hline Non-wheat sequences ${ }^{\mathrm{b}}$ (eliminated) $(n)$ & 558 & 518 \\
\hline \multicolumn{3}{|l|}{ Wheat protein coding sequences } \\
\hline BLASTX, E-value cutoff $1 e^{-3}$ & 96,244 & 59,439 \\
\hline Contigs with a Pfam domain $\left(1 e^{-3}\right)$ & 59,917 & 39,965 \\
\hline Contig sequences without BLASTX $\left(1 e^{-3}\right)$ or Pfam $\left(1 e^{-3}\right)$ & 42,999 & 26,070 \\
\hline \multicolumn{3}{|l|}{ Predicted open reading frames } \\
\hline Predicted ORFs (non-redundant, $>30$ amino acids) & 76,570 & 43,014 \\
\hline Fulllength & 32,548 & 22,868 \\
\hline Missing $5^{\prime}$ end & 26,723 & 12,225 \\
\hline Missing $3^{\prime}$ end & 12,792 & 5,376 \\
\hline Missing $5^{\prime}$ and $3^{\prime}$ end & 4,507 & 2,545 \\
\hline Putative pseudogenes (frameshift and/or premature stop codon) & 9,937 & 5,208 \\
\hline \multicolumn{3}{|l|}{ Putative fused transcripts } \\
\hline Contigs with BLASTX on inconsistent strand & 4,376 & 3,628 \\
\hline Contigs with $>1$ predicted ORFs ( $>30$ amino acids, no repetitive elements, not a pseudogene) & 2,164 & 1,349 \\
\hline Putative fused transcripts (excluding overlaps) ( $n$ ) & 6,409 & 4,866 \\
\hline
\end{tabular}

${ }^{a}$ Open reading frames were predicted with a comparative genomics approach using the findorfprogram and BLASTX alignments (E-value cutoff $1 \mathrm{e}^{-5}$ ) between contigs and proteomes of barley, Brachypodium, rice, maize, sorghum, and Arabidopsis.

${ }^{\mathrm{b}}$ Non-wheat sequences were identified based on taxonomic distribution of top 10 BLASTX hits against $\mathrm{nr}$.

non-protein coding RNAs (lncRNAs) [43,44]. LncRNAs have been shown to regulate a variety of cellular processes and several show increased expression in response to stress and pathogen attack in wheat [45]. The human ENCODE project has demonstrated the value of documenting and storing these non-coding sequences[46].

\section{Pseudogenes}

Using the findorfprogram(Additional file 6)[41] we identified 5,208 ORFs in T. urartu(12.1\%) and 9,937 in T. turgidum(13.0\%) that were disrupted by frameshifts or stop codons (Table 2). Even though the percentages of predicted pseudogenes in these two datasets are relatively close, they are significantly different $(P<0.0001$, Fisher's Exact Test) due to the large sample size. A slightly higher proportion of pseudogenes in T. turgidum than in T. urartu is to be expected since gene duplications are known to lead to relaxed selection [47]. To validate the pseudogene predictions we compared theircodon usage with that of predicted functional genes.Pseudogenecodon usage is expected to drift towards that of intergenic DNA regions due to a lack of purifying selection [48]. Figure 5shows a multidimensional scaling plot of the distances between contigs based on the frequencies of codon usage in ORFs. The partial separation across the two-dimensional space indicates a tendency towards differential codon usage between functional and non-functional ORFs with predicted frameshift mutations or premature stop codons, which provides an independent validation for the pseudogene prediction pipeline. A partial overlap between these two classes is expected for pseudogenes of recent origin.

It is interesting to note that our estimates of the proportion of pseudogenes present in T. urartu and T. turgidumtranscriptomes are lower than the $28 \%^{\mathrm{a}}$ estimate obtained from a T. aestivum transcriptome assembled from Roche 454 reads[21].This discrepancy is not likely to be caused by differences in pseudogene identification methods, since our findorf prediction pipeline estimated a very similar proportion of pseudogenes (27\% of the ORFs) in the recently published transcriptome of $T$. aestivum variety Kukri assembled using a combination of Roche-454 and Illumina GAIIx paired-end reads[27].The higher proportion of pseudogenes observed in T. aestivum than in T. turgidum transcriptomes is unexpected given the short evolutionary time since the origin of T. aestivum from T. turgidum. It is possible that differences in sequencing technologies and/or assembly methods may have also contributed to these differences. For example, homopolymer sequencing errors in Roche 454 sequences generate frameshift mutations, which can result in an overestimation of the proportion of pseudogenes.

\section{Artificially fused transcripts}

During the initial ORF prediction we determined which contigs were aligned to more than one plant protein in the opposite orientation (one BLAST hit to the positive strand, and another to the negative strand). We initially identified a total of 3,628 contigs with inconsistent strands in $T$. urartu $(6.1 \%$ of the total contigs with BLASTX hits) and 4,376 in T. turgidum (4.5\%, Table 2). 


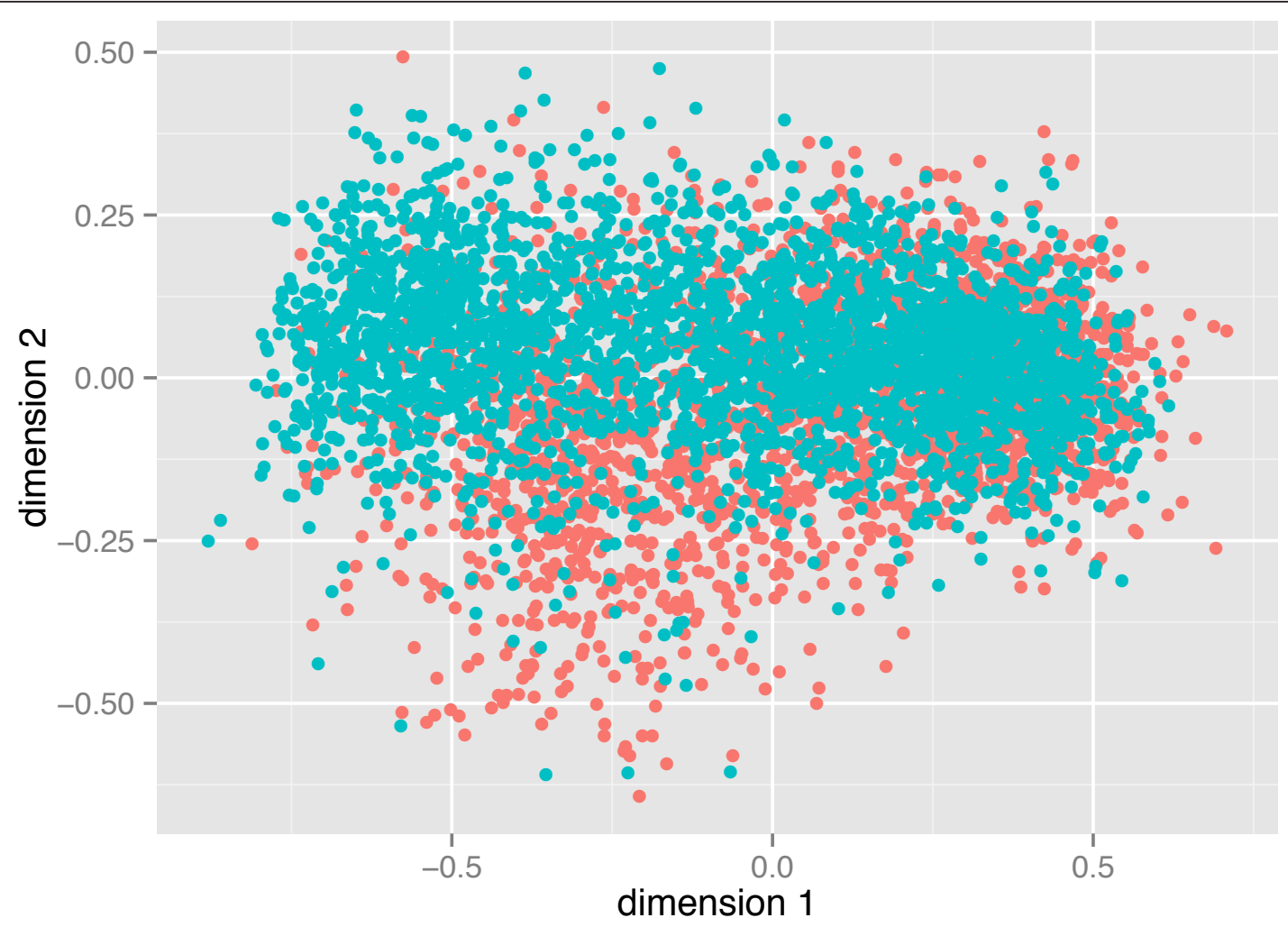

\section{ORF type - frameshift and/or premature stop codon $\bullet$ no interruption}

Figure 5 Comparison of codon usage in predicted genes and pseudogenes. A multidimension scaling scatterplot was generated from a random set of 3,000 full-length and 3,000 pseudogene-containing contigs. Pseudogenes were predicted by findorf by the presence of internal frameshifts or stop codon compared with known plant proteins.

Some of these contigs are likely to be the result of transcripts artificially fused during assembly.

As a complementary method to identify and characterize artificially fused contigs(in both orientations) we performed two consecutive runs of findorf. After the initial ORF prediction we masked the predicted coding region and ran a second round of findorf to identify contigs that include more than one predicted protein. We manually annotated 22 putative fusions (see Additional file 2, Table S4) to characterize their origin and evaluate the predictive value of our strategy. Only six contigs (27.3\%) included ORFs that mapped to different $T$. aestivum genomic contigs[32].For three of them, we were able to identify a common microsatellite in the UTR, a shared inverted region in the UTR, and a common conserved domain as the probable sources of the incorrect fusions (Additional file 2, Table S4). Among the contigs including two ORFs that were mapped to the same genomic contig, five (22.7\%) were fused due to overlapping 3' UTR regions in opposite DNA strands (adjacent genes with opposite orientations). Of the remaining 11 contigs (50\%),the inconsistent ORF prediction was associated with the presence of repetitive regions (three cases), pseudogenes and very short predicted secondary ORFs (five cases), and adjacent ORFs that were incorrectly predicted as separate proteins andrepresentedtrue biological gene fusions (three cases) (Additional file 2 Table S4).

To eliminate incorrectly predicted artificially fused contigs we added additional filters to the prediction of secondary ORFs including: (1) elimination of short predicted ORFs ( $<30$ amino acids);(2) elimination of ORFs predicted as pseudogenes; and (3) elimination of ORFs predicted in regions with significant similarity to repetitive elements (TREP database)[49]. After applying these filters, the number of contigs annotated as putative artificially fused transcripts was reduced byhalf (Table 2). Combining both methods (and excluding overlapping contigs), we estimated a total of 4,866 (8.2\%) and 6,409 (6.7\%) putative artificially fused transcripts for T. urartu and T. turgidum, respectively (Table 2). Predicted fused transcripts are marked either as 'inconsistent_strand' in the comments field or 'predicted_iter2_orf' in the source 
fieldof the GTF annotation files (Supplemental datasets 4 and 5[36]), depending on which of the two methods was used to identify the putative fusion.

In summary, after addition of the secondary ORF predictionsidentified in the artificially fused contigs and the exclusion of predicted pseudogenes, the final transcriptome datasets comprised 37,806 ORFs in T. urartu (Supplemental dataset 4[36]) and 66,633 ORFs in T. turgidum (Supplemental dataset 5[36]). The predicted proteins from these ORF are deposited in Supplemental dataset 6 (T. urartu)and Supplemental dataset 7 (T. turgidum)[36].

\section{Gene structure}

A BLASTN comparison between our transcriptomes and the available genomic sequences for the Chinese Spring chromosome arms [32] allowed us to simultaneously determine gene structure and chromosome location (Supplemental datasets 13 and 14[36]). A threshold of 99\% identity was used to identify the most likely correct homoeolog for each of our predicted ORFs. The analysis of the BLASTN results showed that $46 \%$ of the T. urartu and $55 \%$ of the T. turgidum ORFs have $\geq 99 \%$ identity (and $\geq 65 \%$ coverage) to one or more contigs of Chinese Spring (Table 3). These results indicate that roughly half of our ORFs are represented by the corresponding homoeologous genome in the current genomic assemblies of the wheat chromosome arms, with $40 \%$ in T. urartu and $50 \%$ in T. turgidum being full length ( $>95 \%$ coverage). Significant alignments with the other homoeolog $(94 \% \leq$ Id $<99 \%$, $>65 \%$ coverage) were identified for another $42 \%$ and $33 \%$ of the T. urartu and T. turgidum ORFs, respectively.

These alignments were used to predict gene structure using the program EXONERATE [50]for all the ORFs. We identified complete gene structures ( $>95 \%$ coverage) for $77.6 \%$ of the ORFs and at least partial structures $(>65 \%$ coverage) for $88.0 \%$ of the ORFs (Table 3 weighted averages of the two datasets). The coordinates of the predicted exons are provided in Supplemental Datasets 13 (T. urartu) and 14 (T. turgidum)[36]. These tables also provide percent identities between the predicted ORFs and the Chinese Spring contigs (Table 3) and can be used to infer homoeologs among the T. turgidum ORFs.

\section{Phasing of merged homoeologs to reconstruct genome- specific sub-assemblies}

Based on previous reports [27], we expected that even using very sensitive assemblers, a significant proportion of the homoeologs would be merged creating A/B chimeric contigs (Figure 6A). Therefore, we exploredpostassembly approaches to separate merged contigs. We hypothesized that the separation of two homozygous genomes in a self-pollinated- and therefore, highly homozygous -tetraploid species presents similar challenges to the separation of haplotypes in a sexually reproducing diploid organism. The problem of resolving heterozygous haplotypes from next generation sequencing data has recently been tackled in humans [51] using the HapCUT algorithm[52].

To identify polymorphisms inmerged homoeologs we first re-aligned all the T. turgidum reads back to the assembled contigsallowing a maximum of 10 mismatches per $2 \times 100$ bp paired-end read fragment $(>95 \%$ identity). Using the FreeBayes variant detection program with parameters adjusted for RNA-seq data (see Materials and methods), we identified 1,179,465 polymorphisms, including 958,362 SNPsand 23,424 multinucleotide polymorphisms (MNPs) present in 103,304 contigs (Table 4). There were a total of 74,880 contigs (53.4\%) that contained $>1$ SNP/MNP, which were therefore good candidates for polymorphism phasing (Table 4).As expected, this proportion was much lower in T. urartu, which showed only $25.4 \%$ of the contigs with $>1$ SNP $(21,926 / 86,247)$, which indicates that some close paralogs have been merged in the multi-kmer diploid assembly.

Table 3 Comparison of predicted ORFs (excluding pseudogenes) with $T$. aestivum genomic DNA contigs

\begin{tabular}{lcc}
\hline Transcriptome & T. urartu & T. turgidum \\
\hline Putative correct homoeolog (Id $\geq 99 \%$ identity) & 14,678 & 32,554 \\
$\geq 95 \%$ coverage in one CS contig & 489 & 911 \\
$\geq 95 \%$ coverage in more than one CS contig & 2,094 & 3,136 \\
$\geq 65 \%$ coverage in one or more CS contigs & 12,239 & 17,437 \\
Putative homoeolog from different genome (94\% Id < 99\%) & 1,146 & 1,549 \\
$\geq 95 \%$ coverage in one CS contig & 2,416 & 3,262 \\
$\geq 95 \%$ coverage in more than one CS contig & & 7,370 \\
$\geq 65 \%$ coverage in one or more CS contigs & 4,549 & 414 \\
Not aligned & 195 & 66,633 \\
Id $<94 \%$ or coverage $<65 \%)$ & 37,806 & \\
Number of query sequences with no significant BLAST hits $\left(\mathrm{e}^{-10}\right)$ & \\
\hline Total number of query sequences & & \\
\hline
\end{tabular}


A<smiles>[O-][O+][O-]</smiles>

SNPs in the reads create bubbles in de Bruijn graphs

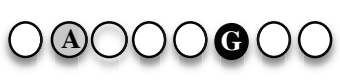

Chimeric assembly
B

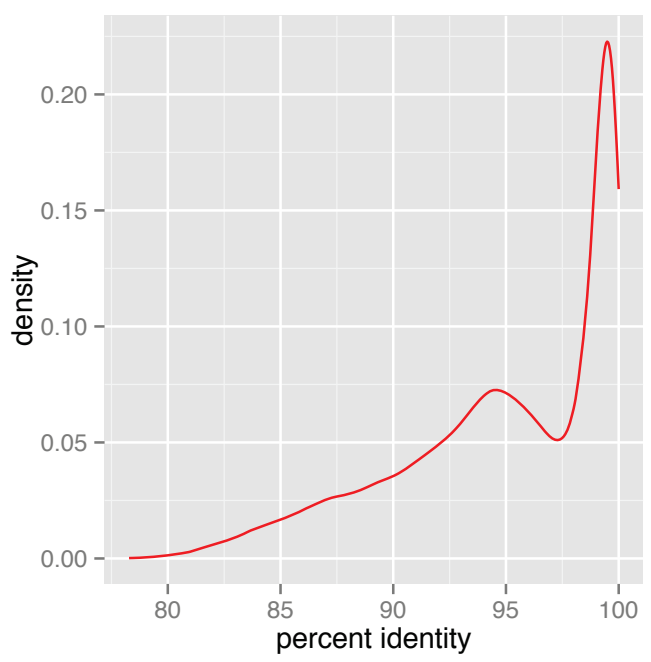

D Contigs with $>1$ SNP/MNPs after phasing

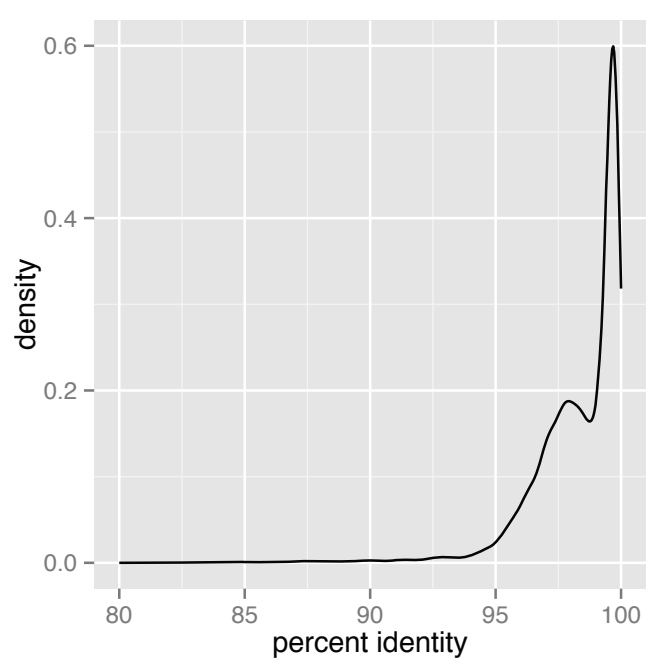

Figure 6 Identification and phasing of A/B contigs merged during the assembly. (A) Schematic illustration of a contig merged during the assembly. Empty circles represent nucleotides that are common between homoeologs. Grey and black circles correspond to biological polymorphisms between homoeologs. (B) Density plots of percent identity between T. turgidum and T. urartufor contigs with <2 SNPs. The 95\% identity peak represents mostly B genome contigs and suggests a relatively good separation of A and B genome contigs in this dataset. (C,D) Density plots of percent identity between T. turgidum and T. urartu for contigs with $\geq 2$ SNPs. (C) Distribution before phasing (note the absence of a bimodal distribution) and (D) after phasing (bimodal distribution as in B).

To test if contigs without SNPs/MNPs were already separated into A/B homoeologs, we plotted the percent identity of twocontig groups (those with and those without SNPs/MNPs) to our T. urartu transcriptome (Figure $6 \mathrm{~B})$. The population of contigs with $<2$ SNPs/MNPs $(65,238$ contigs) showed a bimodal distribution in percent identity corresponding to the predicted distributions of $\mathrm{A} / \mathrm{A}$ and $\mathrm{A} / \mathrm{B}$ homoeologous alignments. This indicates that a large proportion of contigs without SNPs/ MNPs representwell-separated A or B homoeologs. In contrast, the bimodal distribution is not observed in the contigs with $>1$ SNP/MNP (Figure 6C, before phasing), likely due toA/B chimeras with intermediate identity values.

Using the HapCUT program[52](see Materials and methods), wephased $88 \%$ of the SNPs/MNPs detected by FreeBayes in 67,169tetraploid wheat contigs (Table 4), referred to hereafter as reference contigs.Ideally, each reference contig should be phased into two continuous contigs representing the two homoeologs/paralogs. 


\begin{tabular}{|c|c|}
\hline Polymorphisms before phasing & \\
\hline Polymorphisms (n) & $1,179,465$ \\
\hline Singlenucleotide polymorphisms (SNP) (n) & 958,362 \\
\hline Multi-nucleotide polymorphisms (MNP) (n) & 23,42 \\
\hline Insertions & 72,144 \\
\hline Deletions & 39,882 \\
\hline Complex & 84,457 \\
\hline Other (>2 alleles) ${ }^{b}$ & 1,089 \\
\hline \multicolumn{2}{|l|}{ Phasing (HapCUT) } \\
\hline Contigs with <2 SNP/MNP $(n)$ & 65,238 \\
\hline Contigs with >1 SNP/MNP ( $n)$ & 74,880 \\
\hline Phased contigs $(n)$ & 67,169 \\
\hline Phased blocks ( $n$ ) & 81,413 \\
\hline Phased SNPs/MNPs $(n)$ & 864,865 \\
\hline Chimeric reference contigs ( $n$ ) & 34,025 \\
\hline \multicolumn{2}{|l|}{ Readphaser } \\
\hline Reads filtered due to mapping quality $<30(n)$ & $106,003,190$ \\
\hline Reads filtered due to indels ( $n$ ) & $6,544,331$ \\
\hline Reads passed to MIRA ( $n$ ) & $256,016,046$ \\
\hline
\end{tabular}

${ }^{a}$ Complex: composite insertions and substitution events.

${ }^{b}$ Other: includes cases with $>1$ alternative allele.

However, when long stretches without SNPs are present or SNPs cannot be consistently phased, the contig is partitioned into blocks that must be phased independently. In our dataset, roughly $20 \%$ of the contigs were partitioned into more than one block (total 81,413 blocks), resulting in an average of 1.2 blocks per contig. Blocks were classified as being either chimeric (alternating A and B phases within the block) or non-chimeric (all SNPs/MNPs in the same phase). Using this criterion, we established that 34,029 reference contigs ( $51 \%$ of the phased contigs, or $24 \%$ of all contigs)were chimeric and 33,140 (49\% of the phased contigs, or $24 \%$ of all contigs) were non-chimeric. This last set includes cases where only one homoeolog of a close pair was retained in the assembly after CD-HIT.

Because HapCUT generates tables of phased SNPs but not assembled phased sequenceswe developed a new program readphaser (see Materials and methods, Additional file 7and [53]) that sorts the reads within each block into two phases based on the HapCUT tables. Sorted reads are then re-assembled independently bya combination of MIRA [54] and CAP3 (see Materials and methods). To avoid miss-assembly of recent paralogs, readphaser does not include reads where there is evidence of a third valid haplotype.

The MIRA-CAP3 assembly resulted in 244,165 contigs. Since two phases were submitted for each of the 81,413 phased blocks, this result indicates that our readphaserMIRA-CAP3 pipeline further partitionedhalf of the submitted blocks $(81,399)$ into $>1$ contig. To estimate the proportion of sequences from the original contigs that were recovered by the HapCUT-readphaser-MIRA pipeline we aligned the MIRA-CAP3 contigs to the original blocks. These analyses showed that $98 \%$ of the original contigs are represented (at least partially) in the current MIRA-CAP3 assembly, but also that the 244,165 MIRACAP3 contigs cover only $62 \%$ of the original sequences. The sequences not covered include regions of low coverage in the original blocks, long stretches of identical sequences between $\mathrm{A}$ and $\mathrm{B}$ genomes (Figure $1 \mathrm{~B}$ ), and $\mathrm{A} / \mathrm{B}$ SNPs that were not used by HapCUT and readphaser due to low mapping quality values $(<30)$. Reduced mapping quality was particularly prevalent in sequences represented by $>1$ contig with identical regions, such as alternative splicing forms. Because reads derived from these redundant regions can map equally well to multiple locations, their mapping quality is greatly reduced.Roughly $30 \%$ of the reads were excluded from the MIRA assembly due to low mapping quality (Table 4), suggesting that there is a delicate balance between the stringency of the mapping quality and the proportion of phased sequences. Possible alternatives to increase coverage of the phased sequences in the future includeadditional reductions in the reference dataset (for example, alternative splicing forms) or the use of different mapping qualitythresholds for phasing.

To evaluate the quality of the phasing results we usedtwo different approaches. First, we compared the HapCUT phased SNPs for our manuallycurated set of 26 homoeologous gene pairs (Supplemental dataset 1[36]) with their known phases. Before phasing, these 26 gene pairs were represented by 33 contigs with 377 SNPs between the A and B genomes. A comparison of the HapCUT tables and the manually curated genes showed that 372 of 377 SNPs (98.7\%) were correctly phased. Therefore, after phasing only $1.3 \%$ of the SNPs in $24 \%$ of the contigs were still chimeric.Additionally, we compared the phased MIRA assemblies to the T. urartu contigs (A genome, one phase). This approach also showed an overall good separation of homoeologs. Before phasing, the distribution of BLASTN similarity values between T. urartu and unphased reference contigs showed no evidence of a distinct peak for $\mathrm{B}$ genome contigs (Figure $6 \mathrm{C})$. We hypothesize that this is the result of the large proportion of $\mathrm{A} / \mathrm{B}$ chimeric contigs (51\% of the phased contigs with $>1 \mathrm{SNP}$ ), which generate intermediate similarity values that mask the bimodal distribution. In contrast, the alignments generated after phasing show a clear bimodal distribution (Figure 6D). Together, these data indicate that our post-assembly pipeline significantly reduced the number of chimeric transcripts.

\section{Conclusions}

The comparison between our diploid and tetraploidwheat assemblies showed that merging assemblies across 
a wide range of $\mathrm{k}$-mersizeshas a positive effect on de novo transcriptome assemblies in both diploid and polyploid species, but has a larger positive effect on the latter. We speculate that this is related to the heterogeneity in the distribution of SNPs between homoeologs in the different gene classes, which favors full-length assemblies of different genes at different k-mersizes. However, multiple k-mer assemblies also lead to increasesin sequence redundancy, which require post-assembly processing. This is especially challenging in polyploid species where different chimeric contigs can be assembled at different k-mersizes. We showed that a CD-HIT merge using a 95\% identitythreshold, which in wheat includes approximately $95 \%$ of the homoeologous regions, resulted in a good balance between assemblyquality and reduced redundancy.

A critical step in a polyploid transcriptome assembly is the separation of homologs. The approach followed by Schreiber et al. (2012), that implemented a computationally-intensive two-stage assembly using the stringent MIRA assembler in the last step, reduced the proportion of chimeric contigs to $18 \%$ and represented a step in the right direction[27]. Thepost-assembly read phasing pipeline presented in this study represents an advance over current methodsto solve the problem of assembling closely related sequences without generating chimeras.Since this post-assembly pipeline is not dependent on resources specific to wheat, itcouldalso be applied to help resolve similar challenges in assembling transcriptomes ofother homozygous tetraploid species. The only parameter that needs to be adjusted to the level of divergence between the targeted genomes is the maximum number of mismatches allowed in the mapping of the reads back to the contigs for homoeolog SNP discovery.

This specialized bioinformatics pipeline was developed with the main objective to generate a high-quality annotated tetraploid wheat transcriptome. However, some of the new modulesfrom our pipeline, such as readphaser, willlikelyfacilitate development of more general strategiesfor assembling transcriptomes of other tetraploid species.

Comparison of tetraploid wheat contigs with and without phasing indicates that the initial assembly separated well relatively distant homologs (average 95\% identity, see Figure 6B) but failed to distinguish between more closely related sequences (average 97.5\% identity, Figure 6D). A corollary of this interpretation is that only recently originated polyploid species may require phasing for a correct separation of homoeologs.

Our transcriptome annotation effortsyielded a valuable dataset of coding sequences and proteins in wheat that greatly enrichesthe currently sparse wheat proteomic dataset. These integrated datasets are expected to provide valuable references for RNA-seq and proteomics experiments in wheat.We are using this information to develop a gene capture platform for wheat, which is being used in our laboratories to sequence the exome of tetraploid and hexaploid wheat TILLING populations to identify mutations [55].The predicted tetraploid wheat proteome and gene models generated in this study provide a valuable tool for the wheat research community and for those interested in comparative genomic studies including wheat.

\section{Materials and methods}

\section{Plant growth conditions and sample collection}

The diploid wheat T. urartuaccession G1812 was selected for this study due to itsclose relationship to the A genome of hexaploid wheat, availability of aBAC library[56] and ongoing genome sequencing project [57]. The tetraploid wheatT.turgidum Kronos, a modern durum wheat cultivar with high yield potential and excellent pasta quality, was selected based on the availability of mutant TILLING population [55] and the existence of a genome project at Cold Spring Harbor Laboratory[58].

Wheat grain was surface-sterilized in $10 \%$ bleach and incubated at $4^{\circ} \mathrm{C}$ for 2 days before germination. Young root and shoot tissues were collected 2 to 3 weeks after germination. For Kronos only, spike tissue was collected from mature plants at the booting stage and grain tissue was collected 20 days post anthesis.Sampleswere immediately frozen in liquid nitrogenand stored at $-80^{\circ} \mathrm{C}$.

\section{Benchmark gene sets}

To test the quality of our assembly pipeline, we used two wheat benchmark sets. The first set consisted of 13,472 full-length non-redundant $T$. aestivum cDNA clones from the TriticeaeFull-Length CDS DataBase sequenced by traditional Sanger technology [35]. The second dataset consisted of 52wheat genes (26 homoeolog pairs) previously assigned to either the A or B genomes and annotated for gene structure in our laboratory (Supplemental dataset1[36]).

\section{Library construction and sequencing}

Total RNA was extracted using Spectrum ${ }^{\mathrm{TM}}$ Plant Total RNA Kit (Sigma), from which mRNA was purified using the Dynabeads mRNA Purification kit (Illumina) and transcribed to cDNA using SuperScript II Reverse transcriptase (Invitrogen) and DNA Polymerase I (Promega). cDNA was purified using the PCR purification kit (Qiagen) and quality was assessed on the Bioanalyzer machine. The cDNAs were fragmented in a Covaris machine $(10 \%$ duty cycle, Intensity: 4, Cycles per burst: 200,80 s), treated with end-repair enzymes: T4 DNA polymerase (Invitrogen), Klenow DNA Polymerase (Invitrogen), and T4 Polynucleotide kinase (Invitrogen), and adenylated with Klenow exo (3' to 5' exo minus) (Invitrogen). Illumina PE 
adapters (Illumina Multiplexing kit, PE-400-1001) were ligated using the Quick Ligation kit (New England Biolabs) and purified with the minElute kit (Qiagen). DNA fragments were separated on a $2 \%$ agarose TAE gel; fragment with average sizes of 300, 400, 500, and 600 bp were extracted using Gel Excision tips (Gel Company) and purified using the Gel Extraction kit (Qiagen). Libraries were multiplexed according to the instructions in the Illumina Multiplexing kit (Illumina) with 12 cycles of PCR amplification. Final libraries were purified using Ampure beads in a 1:1 sample volume to bead volume ratio (Ampure). The quality of the libraries was assessed on the Bioanalyzer machine using High Sensitivity DNA kit reagents (Agilent).

Library normalization was performed using double stranded DNA nuclease (Evrogen) as published before[59]. Four 300-bp libraries from roots, shoots, spike and grain were pooled for normalization. A total of $250 \mathrm{ng}$ of DNA was allowed to hybridize for $5 \mathrm{~h}$ at $68^{\circ} \mathrm{C}$ in either $\mathrm{NaCl}$ or TMAC buffer, equilibrated for $10 \mathrm{~min}$ at $68^{\circ} \mathrm{C}$ in DSN buffer, and digested with $1 \mu \mathrm{L}$ of DSN enzyme for $25 \mathrm{~min}$ at $68^{\circ} \mathrm{C}$. A 'no DSN enzyme' control was processed simultaneously to access the normalization efficiency. All samples were re-amplified with 10 to 12 PCR cycles.

All libraries were sequenced using the $100 \mathrm{bp}$ pairedend protocol on four lanes of Illumina HiSeq2000 machines at the University of California Davis (UCD) Genome Center. Base quality calls and demultiplexing was done with the CASAVA 1.8.0 pipeline (Illumina).

\section{Transcriptome assembly}

Overall read quality was assessed using the $\mathrm{R}$ package qrqc [60]. Illumina adapter sequences were trimmed with the program Scythe v. 0.981[61](-p 0.2-n 3) and poor quality bases were trimmed with Sickle v. 1.2[62] $(-\mathrm{q}=20)$. Reads arising from common contaminants, including Homo sapiens and Escherichia coli DNA, wheat mitochondrial and chloroplast sequences as well as wheat rRNA (Additional file 2, Table S3) were identified using BLAT v.34 [63] with the default parameters and then removed.

Artificial sample variation (differences in gene coverage in RNA-seq) and k-mersequencesincluding sequencing errors were removed prior to the assembly using a digital normalization algorithm [33] (normalize-by-median.py -C 20 -k 20 -N 4 -x 2e9). A previously constructed wheat RNA-seq library (SRA ERX022241)[34] was used to assess and compare the quality of the assembly before and after normalization as well as to determine optimal parameters for the assembly.

Within each species, combined reads from the different libraries were assembled with CLC Genomics Workbench v. 5.5de novo assembly algorithm. Initially, we tested several de novo assembly algorithms including Trinity and Oases, and we chose to use CLC due to its performance on the benchmark full-length wheat cDNA datasets and overall assembly parameters. Paired-end distances were specified for each library based on preliminary mapping experiments against benchmark full-length wheat cDNA sequences. Ten individual assemblies were constructed at variable k-mers (word size of 21, 25, 31, 35, 41, 45, 51, 55, $61,63)$. A word size of 64 is the maximum permitted when using CLC version 5.5. Other parameters included: bubble size $=400$, read mapping $=$ global, and 95\% similarity which were chosen based on optimizations using a small read set and the 13,472 full-length wheat cDNA benchmark data.

The individual $\mathrm{k}$-mer assemblies were concatenated and redundancy was reduced using CD-HIT v.4.5.4 [64]. Contig merging was carried out at $95 \%$ identity level for T. turgidum (cd-hit-est -r 1 -c 0.95 -n 8 -T 0 -gap -2) and $99 \%$ identity level for T.urartu (cd-hit-est $-\mathrm{r} 1$-c 0.99-n 8 -T 0 -gap -2).

To reconstruct genes partially assembled at different kmer lengths, we implemented a protein-guided assembly approach, blast2cap3 [39](Additional file 5). Contigs were first clustered based on a common top BLASTX [65] hit (E-value cutoff $1 \mathrm{e}^{-3}$ ) against T. aestivum, Hordeum vulgare, Brachypodium distachyon, Oryza sativa, Sorghum bicolor, Zea mays, and Arabidopsis thaliana protein datasets (Additional file 2, Table S3).Each contig cluster sharing a common protein hit was passed to the overlap-based assembly program CAP3 [40] (cap3 -p 99 -k 0 -o 100).

To identify contaminating sequences from non-wheat organisms (for example, plant epiphytes and pathogens), we used the following taxonomy-based pipeline at the post-assembly stage. First, all contigs were passed through BLASTX against the NCBI non-redundant (nr) database, retaining the top 10 hits using an E-value cutoff of $1 \mathrm{e}^{-10}$. The kingdom-level taxonomy of all hits was retrieved from NCBI's taxonomy data structure using an adapted publicly-available Bioperl script (bp_classify_by_kingdom. $\mathrm{pl}[66])$. Sequences with all top 10 hits that matched nonplant organisms were considered likely contaminants and were removed from the assembly using custom Perl scripts.

\section{ORF prediction and functional annotation}

We developed the program findorf to predict ORFs and pseudogenes(Additional file 6) [41].Findorf relies upon BLASTX alignments against protein databasesand includes subcommands: 'findorf join' and 'findorf predict'.The results from BLASTX searches (E-value cutoff $1 \mathrm{e}^{-3}$ ) against plant databases (Additional file 2, Table S3) and HMMER3.0 scans of all contigs translated in all six open reading framesagainst Pfam-A (hmmscan e-value $1 \mathrm{e}^{-3}$-domE 1 -noali) were passed to 'findorf join' (-domain-hits) and ORFs were predicted using 'findorf predict' (-evalue $1 \mathrm{e}^{-5}$-verbose -use-pfam). 
Findorf uses a conservative approach to identify translation startsites (TSS) and if an additional methionine exists $5^{\prime}$ of the predicted TSS, the information is provided in the GTF files (Supplemental datasets 4 and 5 [36]). In cases where HSPs disagree on frame across a majority of alignments, the contig is annotated as having a majority frameshift and the frame of the 5'-most HSP is used during this initial ORF prediction. In cases when findorf detects significant HSPs in opposite strands, it annotates the contig as 'inconsistent strand' and outputs no ORF.

Functional annotation of predicted proteins was done using Hidden-Markov Model based searches against Pfam-A database[42]implemented in HMMER3.0 [67] (hmmscan e-value $1 \mathrm{e}^{-3}-$ domE 1 -noali). Candidate repetitive elements and transposons were identified based on results from BLASTN and BLASTX searches against the nucleotide and protein Triticeae Repeat Sequence Databases (TREP)[49] using an E-value cutoff of $1 \mathrm{e}^{-10}$.

\section{Identification of pseudogenes and codon bias analyses}

A contig was identified as a putative pseudogeneby findorf when a significant protein alignment (BLASTX, E-value $1 \mathrm{e}^{-5}$ ) between contig sequence and related protein sequences (Additional file 2, Table S3)was disrupted by a premature stop codon or includeda frameshift mutation.In the first case, a significant HSP overlapping the related protein sequenceby $>20$ amino acids beyond the predicted premature stop codon was required to annotate the contig sequence as a putative pseudogene. In the second case, a contig was annotated as having a frameshift mutation if the HSPs matched different sections of the same reference proteins in two different frames on the same strand.

To further characterize the predicted pseudogenes, we compared codon usage between samples of 3,000 pseudogenes and 3,000 genes that did not include premature stops or frameshifts. Frequencies of codonswere converted to proportions, and Manhattan distances were calculated among the 6,000 data points. To visualize these results, we used a metric multidimensional scaling approach, implemented inthe $\mathrm{R}$ language.

\section{Identification of artificially fused transcripts ( $<1$ ORF)}

During the initial ORF prediction, a strand consistency filter was imposed to identify contigs with any BLASTX hits on opposite DNA strands, which gave an estimate of putative merged transcripts. In addition, we used an iterative ORF prediction to identify presence of secondary ORFs. The first iteration of BLASTX alignments (E-value $1 \mathrm{e}^{-3}$ cutoff) were masked and the masked sequences were run a second time through findorf with the same parameters.
After manual inspection of the initial results, we imposed additional filtering criteria to identify artificially fused transcripts, including the exclusion of pseudogenes and repetitive elements as well as very short ORFs $(<30$ amino acids).

\section{Predicting gene exons and assigning genes to chromosome arms}

A BLASTN search with an E-value cutoff of $1 \mathrm{e}^{-10}$ was performed between our T. turgidum transcriptome and the genomic sequences of the individual chromosome arms of Chinese Spring generated by the IWGSC[32]. A Perl script was written to process the BLAST output. A hit - tagged with the name of the chromosome arm was stored if it shared on average $\geq 94 \%$ across all HSPs and was stored together with other contigs that hit the exact same chromosome arm. If the hits to each arm covered $\geq 65 \%$ of the ORF length and matched one or more Chinese Spring contigs, a gene exon-intron prediction model was created with EXONERATE v.2.2.0 $[50,68]$ (-model est2genome -ryo).

\section{Phasing SNPs from different homoeologs}

To generate genome-specific assemblies in tetraploid wheat, we first aligned $T$. turgidum reads with the T. turgidum reference transcriptome (140,118 contigs) using Novoalign software (v. 2.08.01; -F ILM1.8 -o SAM -o Sync -i PE -r Random -t 300) and insert size range specific to each library (see Table 1). We compared bowtie, bwa, and Novoalign and selected the latter because it maximized our quality control parameters (most reads aligned and most reads aligned in proper pairs). Polymorphisms among the mapped readswere detectedusing the FreeBayes software[69](v.0.9.6; parameters:-p 2 -k -min-alternate-count 2 -p 2 -min-coverage 4 -T 0.05) as it has been shown to perform well on RNA-seq data [70]. Called SNPs and MNPs were phased using the HapCUTv.0.5software[52] with default parameters. All phased SNPs are reported in HapCUT tabular format in Supplemental dataset 11 [36].

\section{Assembling phased reads into homoeolog-specific sequences}

To generate homoeolog-specific sub-assemblies we tested three different strategies. First, we tried to use the initial reference contig and replace the phased SNPs. However, the presence of non-phased SNPs due to low mapping quality and indels resulted in residual chimeric sequences. Second we attempted to reconstruct consensus sequences from the phased reads based on mapping positions relative to the reference contig, but the presence of indels between the A and B genomes (particularly in UTR regions) complicated the correct reconstruction of consensus sequences. 
Finally, we were successful in reconstructing homoeologspecific sub-assemblies by sorting the reads within each phased SNP block based on the HapCUT output, and de novo re-assembling the reads for each block and phase using parallelized runs ofMIRA assembler [54].

To sort the reads by phase we developed the program readphaser (Additional file 7)[53]. Readphaser extracts reads that include haplotype-specific SNPs identified by HapCUT and separates them into two phased sets that are independently passed to MIRA. Readphaser filters reads with low mapping quality $(\mathrm{mq}<30)$,optical or PCR duplicates, or containing indels. Reads containing out of phase variants, due to sequencing error, tri-allelic variants, or more than two real phases (for example, recent duplications) were placed into an additional set of reads that were unused during assembly. Since some out of phase variants may be biologically interesting, readphaser outputs an additional file with the inconsistent variants in reads.

Re-assembly of sorted reads was performed using a custom Perl script created to run parallel instances of MIRA v. 3.2.1[54] (parameters -job=denovo,est,Solexa, padded option) on multiple cores. CAP3 [40](using default parameters) was then run with the MIRA contigs generated for each phasing block to further extend the assemblies. To evaluate the coverage of the phased contigs assembled by MIRA, we aligned sequences back to their original contig from our reference transcriptome assembly with global-local alignments of both the forward and reverse complement using the function pairwiseAlignment in the Bioconductor package Biostrings[71]. Alignments with scores $<10$ (gap open penalty $=-8$, gap extension penalty $=-2$ ) were not considered. Assembly coverage was calculated using the coverage function in the Bioconductor package IRanges[72].

\section{Data access}

The data from this study is linked to the BioProject PRJNA191053 established for T. urartu and Bioproject PRJNA191054 for T. turgidum. Raw data is available at the Short Read Archive (accession numbers: SRR769749, SRR769750, SRR863375, SRR863376, SRR863377, SRR863384, SRR863385, SRR863386, SRR863387, SRR863389, SRR863390, SRR863391, SRR863394). Filtered contigs are avaialbe through the TSA archive under accession numbers GAKL00000000 for T. urartu and GAKM00000000 for T. turgidum. All supplemental datasets can also be accessed atthe Project Website [36]. A public BLAST site is available at the public USDA GrainGenes database[73].

\section{Endnotes}

a Originally published as $38 \%$ but corrected recently to $28 \%$

\section{Additional material}

Additional file 1: Members of the International Wheat Sequencing
Consortium
Additional file 2: Supplemental Tables (Tables S1-S4)
Additional file 3: Supplemental Figure S1
Additional file 4: Supplemental Figure S2
Additional file 5: blast2cap3 program
Additional file 6: findorf program
Additional file 7: readphaser program

\section{Abbreviations}

BLAST: Basic Local Alignment Search Tool; DSN: DoublestrandDNAnuclease; EMS: Ethyl Methanesulfonate; HMM: Hidden Markov Model; HSP: HighScoringSegmentPair; IWGSC:

InternationalWheatGenomeSequencingConsortium; IncRNAs: longnonproteincodingRNAs; LRR: LeucineRichRepeats; MNP: Multiple Nucleotide Polymorphism; NBS: NucleotideBindingSite; ORF: Open Reading Frame; SNP: Single Nucleotide Polymorphism;SRA: ShortReadArchive; TSA:

TranscriptomeShotgunAssembly; TILLING:

TargetinglnducedLocalLesionsinGenomes;TSS: TranslationStartSite; UTR: UntranslatedRegion.

\section{Competing interests}

The authors declare that they have no competing interests.

\section{Authors' contributions}

KVK: RNA-seq library preparation, bioinformatics pipeline design, transcriptome assembly and annotation,analyses of the disease resistance gene family, SNP detection, and SNP phasing;VB:bioinformatics software development(blast2cap3, findorf, and readphaser), data analysis, and visualization; PB, SA, CU: gene model prediction; EA and SW: MIRA assemblies;SP, FT, MS, JD:curation team (benchmark genes and quality control). IWGSC contributed the unpublished assemblies of the genomic sequences of the Chinese Spring chromosome arms.KVK and JD designed the study and wrote the first draft of the manuscript. All authors participated in data analyses, contributed to writing andcritical evaluation of the manuscript.

\section{Acknowledgements}

This work has been funded by support provided to JD by the Howard Hughes Medical Institute and the Gordon and Betty Moore Foundation (grant number GBMF3031) and in part by the National Research Initiative Competitive Grants no. 2011-68002-30029 and 2011-67013-30077 from the USDA National Institute of Food and Agriculture. CU acknowledges support from Biotechnology and Biological Sciences Research Council (BBSRC) to CU and SA (grant BB/J003557/1). KVK has been supported by USDA NIFA postdoctoral fellowship grant number 2012-67012-19811. Authors are grateful to Vikas Bansal (Scripps Translational Science Institute) for his advice on the HapCUT program, to Rachel Brem (University of California, Berkeley) and Chris Ellison (University of California, Berkeley) for advice on SNP detection in RNA-seq data, to the Novocraft support team advising on read mapping, and to the University of California Davis (UCD) Genome Center for excellent sequencing data. The assemblies of non-repetitive genomic regions of the Chinese Spring chromosome arms used in the gene model predictions were obtained in the framework of the International Wheat Genome Sequencing Consortium and the Survey Sequencing Initiative. A list of institutions of the International Wheat Genome Sequencing Consortium can be found in Additional File 1.

\section{Authors' details}

${ }^{1}$ Dept. Plant Sciences, University of California, Davis, CA 9561, USA. ${ }^{2}$ The Genome Analysis Centre, Norwich Research Park, Norwich NR4 7UH, UK. ${ }^{3}$ Microbiology, University of Buenos Aires, INBA-CONICET, Buenos Aires, Argentina. ${ }^{4}$ Department of Plant Pathology, Kansas State University, 
Manhattan, KS 66506, USA. ${ }^{5}$ International Wheat Genome Sequencing Consortium. ${ }^{6} \mathrm{John}$ Innes Centre, Norwich Research Park, Norwich NR4 7UH, UK. ${ }^{7}$ Howard Hughes Medical Institute, Chevy Chase, MD 20815, USA.

Received: 25 May 2013 Revised: 7 June 2013 Accepted: 25 June 2013 Published: 25 June 2013

\section{References}

1. Doyle JJ, Flagel LE, Paterson AH, Rapp RA, Soltis DE, Soltis PS, Wendel JF: Evolutionary genetics of genome merger and doubling in plants. Annu Rev Genet 2008, 42:443-461.

2. Soltis PS, Soltis DE: The role of hybridization in plant speciation. Annu Rev Plant Biol 2009, 60:561-588.

3. Blanc $\mathrm{G}$, Wolfe $\mathrm{KH}$ : Widespread paleopolyploidy in model plant species inferred from age distributions of duplicate genes. Plant Cell 2004, 16:1667-1678.

4. Dubcovsky J, Dvorak J: Genome plasticity a key factor in the success of polyploid wheat under domestication. Science 2007, 316:1862-1866.

5. Bowers JE, Chapman BA, Rong J, Paterson AH: Unravelling angiosperm genome evolution by phylogenetic analysis of chromosomal duplication events. Nature 2003, 422:433-438.

6. Paterson $\mathrm{AH}$, Bowers JE, Chapman BA: Ancient polyploidization predating divergence of the cereals, and its consequences for comparative genomics. Proc Natl Acad Sci USA 2004, 101:9903-9908.

7. Dvorak J, Zhang HB: Variation in repeated nucleotide sequences sheds light on the phylogeny of the wheat B and G genomes. Proc Natl Acad Sci USA 1990, 87:9640-9644.

8. Dvorak J, Terlizzi P, Zhang HB, Resta P: The evolution of polyploid wheats: identification of the A genome donor species. Genome 1993, 36:21-31.

9. Daud HM, Gustafson JP: Molecular evidence for Triticum speltoides as a Bgenome progenitor of wheat (Triticum aestivum). Genome 1996, 39:543-548.

10. Huang S, Sirikhachornkit A, Su X, Faris J, Gill B, Haselkorn R, Gornicki P: Genes encoding plastid acetyl-CoA carboxylase and 3-phosphoglycerate kinase of the Triticum/Aegilops complex and the evolutionary history of polyploid wheat. Proc Natl Acad Sci USA 2002, 99:8133-8138.

11. Choulet F, Wicker T, Rustenholz C, Paux E, Salse J, Leroy P, Schlub S, Le Paslier MC, Magdelenat G, Gonthier C, Couloux A, Budak H, Breen J, Pumphrey M, Liu S, Kong X, Jia J, Gut M, Brunel D, Anderson JA, Gill BS, Appels R, Keller B, Feuillet C: Megabase level sequencing reveals contrasted organization and evolution patterns of the wheat gene and transposable element spaces. Plant Cell 2010, 22:1686-1701.

12. Wicker T, Yahiaoui N, Keller B: Contrasting rates of evolution in Pm3 loci from three wheat species and rice. Genetics 2007, 177:1207-1216.

13. Isidore E, Scherrer B, Chalhoub B, Feuillet C, Keller B: Ancient haplotypes resulting from extensive molecular rearrangements in the wheat $A$ genome have been maintained in species of three different ploidy levels. Genome Res 2005, 15:526-536.

14. Leister D: Tandem and segmental gene duplication and recombination in the evolution of plant disease resistance gene. Trends Genet 2004, 20:116-122.

15. Cantu D, Vanzetti LS, Sumner A, Dubcovsky M, Matvienko M, Distelfeld A, Michelmore RW, Dubcovsky J: Small RNAs, DNA methylation and transposable elements in wheat. BMC Genomics 2010, 11:408.

16. Dvorak J, Yang ZL, You FM, Luo MC: Deletion polymorphism in wheat chromosome regions with contrasting recombination rates. Genetics 2004, 168:1665-1675.

17. Akhunov ED, Akhunova AR, Linkiewicz AM, Dubcovsky J, Hummel D, Lazo G, Chao S, Anderson OD, David J, Qi L, Echalier B, Gill BS, Gustafson JP, La Rota M, Sorrells ME, Zhang D, Nguyen HT, Kalavacharla V, Hossain K, Kianian SF, Peng J, Lapitan NL, Wennerlind EJ, Nduati V, Anderson JA, Sidhu D, Gill KS, McGuire PE, Qualset CO, et al: Synteny perturbations between wheat homoeologous chromosomes caused by locus duplications and deletions correlate with recombination rates. Proc Natl Acad Sci USA 2003, 100:10836-10841.

18. Feldman M, Levy AA: Genome evolution due to allopolyploidization in wheat. Genetics 2012, 192:763-774.

19. Brenchley R, Spannagl M, Pfeifer M, Barker GL, D'Amore R, Allen AM, McKenzie N, Kramer M, Kerhornou A, Bolser D, Kay S, Waite D, Trick M, Bancroft I, Gu Y, Huo N, Luo MC, Sehgal S, Gill B, Kianian S, Anderson O, Kersey P, Dvorak J, McCombie WR, Hall A, Mayer KF, Edwards KJ, Bevan MW,
Hall N: Analysis of the bread wheat genome using whole-genome shotgun sequencing. Nature 2012, 491:705-710.

20. Wicker T, Mayer KF, Gundlach H, Martis M, Steuernagel B, Scholz U, Simkova H, Kubalakova M, Choulet F, Taudien S, Platzer M, Feuillet C, Fahima T, Budak H, Dolezel J, Keller B, Stein N: Frequent gene movement and pseudogene evolution is common to the large and complex genomes of wheat, barley, and their relatives. Plant Cell 2011, 23:1706-1718.

21. Akhunov ED, Sehgal $S$, Liang $H$, Wang $S$, Akhunova AR, Kaur G, Li W, Forrest KL, See D, Simkova H, Ma Y, Hayden MJ, Luo M, Faris JD, Dolezel J, Gill BS: Comparative analysis of syntenic genes in grass genomes reveals accelerated rates of gene structure and coding sequence evolution in polyploid wheat. Plant Physiol 2013, 161:252-265.

22. Paux E, Roger D, Badaeva E, Gay G, Bernard M, Sourdille P, Feuillet C: Characterizing the composition and evolution of homoeologous genomes in hexaploid wheat through BAC-end sequencing on chromosome 3B. Plant J 2006, 48:463-474.

23. Schulz MH, Zerbino DR, Vingron M, Birney E: Oases: robust de novo RNAseq assembly across the dynamic range of expression levels. Bioinformatics 2012, 28:1086-1092.

24. Grabherr MG, Haas BJ, Yassour M, Levin JZ, Thompson DA, Amit I, Adiconis X, Fan L, Raychowdhury R, Zeng Q, Chen Z, Mauceli E, Hacohen N, Gnirke A, Rhind N, di Palma F, Birren BW, Nusbaum C, Lindblad-Toh K, Friedman N, Regev A: Full-length transcriptome assembly from RNA-Seq data without a reference genome. Nat Biotechnol 2011, 29:644-652.

25. Robertson G, Schein J, Chiu R, Corbett R, Field M, Jackman SD, Mungall K, Lee S, Okada HM, Qian JQ, Griffith M, Raymond A, Thiessen N, Cezard T, Butterfield YS, Newsome R, Chan SK, She R, Varhol R, Kamoh B, Prabhu AL, Tam A, Zhao Y, Moore RA, Hirst M, Marra MA, Jones SJ, Hoodless PA, Birol I: De novo assembly and analysis of RNA-seq data. Nat Methods 2010, 7:909-912.

26. Gruenheit N, Deusch O, Esser C, Becker M, Voelckel C, Lockhart P: Cutoffs and k-mers: implications from a transcriptome study in allopolyploid plants. BMC Genomics 2012, 13:92.

27. Schreiber AW, Hayden MJ, Forrest KL, Kong SL, Langridge P, Baumann U: Transcriptome-scale homoeolog-specific transcript assemblies of bread wheat. BMC Genomics 2012, 13:492.

28. Li C, Dubcovsky J: Wheat FT protein regulates VRN1 transcription through interactions with FDL2. Plant J 2008, 55:543-554.

29. Duan J, Xia C, Zhao G, Jia J, Kong X: Optimizing de novo common wheat transcriptome assembly using short-read RNA-Seq data. BMC Genomics 2012, 13:392.

30. Cantu D, Pearce SP, Distelfeld A, Christiansen MW, Uauy C, Akhunov E, Fahima T, Dubcovsky J: Effect of the down-regulation of the high Grain Protein Content (GPC) genes on the wheat transcriptome during monocarpic senescence. BMC Genomics 2011, 12:492.

31. Paux E, Sourdille P, Salse J, Saintenac C, Choulet F, Leroy P, Korol A, Michalak M, Kianian S, Spielmeyer W, Lagudah E, Somers D, Kilian A, Alaux M, Vautrin S, Berges H, Eversole K, Appels R, Safar J, Simkova H, Dolezel J, Bernard M, Feuillet C: A physical map of the 1-gigabase bread wheat chromosome 3B. Science 2008, 322:101-104.

32. International Wheat Genome Sequencing Consortium. [http://www. wheatgenome.org].

33. Brown C, Howe A, Zhang Q, Pyrkosz A, Brom T: A Reference-Free Algorithm for Computational Normalization of Shotgun Sequencing Data. 2012 [http://arxiv.org/abs/1203.4802], arXiv.

34. Trick M, Adamski NM, Mugford SG, Jiang CC, Febrer M, Uauy C: Combining SNP discovery from next-generation sequencing data with bulked segregant analysis (BSA) to fine-map genes in polyploid wheat. BMC Plant Biol 2012, 12:14.

35. Mochida K, Yoshida T, Sakurai T, Ogihara Y, Shinozaki K: TriFLDB: a database of clustered full-length coding sequences from Triticeae with applications to comparative grass genomics. Plant Physiol 2009, 150:1135-1146.

36. Project website accompanying this paper: $T$. turgidum and T. urartu files for contigs, open reading frames, predicted proteins and gene models. [http://maswheat.ucdavis.edu/Transcriptome/index.htm].

37. Lo C, Bashir A, Bansal V, Bafna V: Strobe sequence design for haplotype assembly. BMC Bioinformatics 2011, Suppl 1:S24.

38. Haznedaroglu BZ, Reeves D, Rismani-Yazdi H, Peccia J: Optimization of de novo transcriptome assembly from high-throughput short read 
sequencing data improves functional annotation for non-model organisms. BMC Bioinformatics 2012, 13:170.

39. Buffalo V: Blast2cap3 software. [https://github.com/vsbuffalo/blast2cap3]

40. Huang X, Madan A: CAP3: A DNA sequence assembly program. Genome Res 1999, 9:868-877.

41. Buffalo V: Findorf software. [https://github.com/vsbuffalo/findorf].

42. Finn RD, Mistry J, Tate J, Coggill P, Heger A, Pollington JE, Gavin OL, Gunasekaran P, Ceric G, Forslund K, Holm L, Sonnhammer EL, Eddy SR, Bateman A: The Pfam protein families database. Nucleic Acids Res 2010 38:D211-222.

43. Carninci P, Kasukawa T, Katayama S, Gough J, Frith MC, Maeda N, Oyama R, Ravasi T, Lenhard B, Wells C, Kodzius R, Shimokawa K, Bajic VB, Brenner SE, Batalov S, Forrest AR, Zavolan M, Davis MJ, Wilming LG, Aidinis V, Allen JE, Ambesi-Impiombato A, Apweiler R, Aturaliya RN, Bailey TL, Bansal M, Baxter L, Beisel KW, Bersano T, Bono H, et al: The transcriptional landscape of the mammalian genome. Science 2005, 309:1559-1563.

44. Derrien $T$, Johnson R, Bussotti $G$, Tanzer A, Djebali S, Tilgner $H$, Guernec $G$, Martin D, Merkel A, Knowles DG, Lagarde J, Veeravalli L, Ruan X, Ruan Y, Lassmann T, Carninci P, Brown JB, Lipovich L, Gonzalez JM, Thomas M, Davis CA, Shiekhattar R, Gingeras TR, Hubbard TJ, Notredame C, Harrow J, Guigo R: The GENCODE v7 catalog of human long noncoding RNAs: analysis of their gene structure, evolution, and expression. Genome Res 2012, 22:1775-1789.

45. Xin $M$, Wang $Y$, Yao $Y$, Song $N, H u$ Z, Qin D, Xie C, Peng H, Ni Z, Sun Q: Identification and characterization of wheat long non-protein coding RNAs responsive to powdery mildew infection and heat stress by using microarray analysis and SBS sequencing. BMC Plant Biol 2011, 11:61.

46. Harrow J, Frankish A, Gonzalez JM, Tapanari E, Diekhans M, Kokocinski F, Aken BL, Barrell D, Zadissa A, Searle S, Barnes I, Bignell A, Boychenko V, Hunt T, Kay M, Mukherjee G, Rajan J, Despacio-Reyes G, Saunders G, Steward C, Harte R, Lin M, Howald C, Tanzer A, Derrien T, Chrast J, Walters N, Balasubramanian S, Pei B, Tress M, et al: GENCODE: the reference human genome annotation for The ENCODE Project. Genome Res 2012, 22:1760-1774.

47. Ohno S: Evolution by Gene Duplication New York: Springer-Verlag; 1970.

48. Echols N, Harrison P, Balasubramanian S, Luscombe NM, Bertone $P$, Zhang Z, Gerstein M: Comprehensive analysis of amino acid and nucleotide composition in eukaryotic genomes, comparing genes and pseudogenes. Nucleic Acids Res 2002, 30:2515-2523.

49. Wicker T, Matthews DE, Keller B: TREP: a database for Triticeae repetitive elements. TRENDS in Plant Science 2002, 7:561-562.

50. Slater GS, Birney E: Automated generation of heuristics for biological sequence comparison. BMC Bioinformatics 2005, 6:31.

51. Kitzman JO, Mackenzie AP, Adey A, Hiatt JB, Patwardhan RP, Sudmant PH, $\mathrm{Ng} \mathrm{SB}$, Alkan C, Qiu R, Eichler EE, Shendure J: Haplotype-resolved genome sequencing of a Gujarati Indian individual. Nat Biotechnol 2011, 29:59-63.

52. Bansal V, Bafna V: HapCUT: an efficient and accurate algorithm for the haplotype assembly problem. Bioinformatics 2008, 24:1153-159.

53. Buffalo V: Readphaser - separate reads based on mapping results and HapCUT data.[https://github.com/vsbuffalo/readphaser].

54. Chevreux B, Pfisterer T, Drescher B, Driesel AJ, Muller WE, Wetter T, Suhai S: Using the miraEST assembler for reliable and automated mRNA transcript assembly and SNP detection in sequenced ESTs. Genome Res 2004, 14:1147-1159.

55. Uauy C, Paraiso F, Colasuonno P, Tran RK, Tsai H, Berardi S, Comai L, Dubcovsky J: A modified TILLING approach to detect induced mutations in tetraploid and hexaploid wheat. BMC Plant Biol 2009, 9:115.

56. Akhunov ED, Akhunova AR, Dvorak J: BAC libraries of Triticum urartu, Aegilops speltoides and Ae. tauschii, the diploid ancestors of polyploid wheat. Theor Appl Genet 2005, 111:1617-1622.

57. Ling $H Q$, Zhao S, Liu D, Wang J, Sun H, Zhang C, Fan H, Li D, Dong L, Tao Y, Gao C, Wu H, Li Y, Cui Y, Guo X, Zheng S, Wang B, Yu K, Liang Q, Yang W, Lou X, Chen J, Feng M, Jian J, Zhang X, Luo G, Jiang Y, Liu J, Wang $Z$, Sha $Y$, et al: Draft genome of the wheat A-genome progenitor Triticum urartu. Nature 2013, 496:87-90.

58. Wheat Genome Sequencing Project. [http://www.cshl.edu/genome/ wheat].

59. Matvienko M, Kozik A, Froenicke L, Lavelle D, Martineau B, Perroud B, Michelmore $R$ : Consequences of normalizing transcriptomic and genomic libraries of plant genomes using a duplex-specific nuclease and tetramethylammonium chloride. PLoS One 2013, 8:e55913.
60. Buffalo V: Quick Read Quality Control. [http://bioconductor.org/packages/ 2.11/bioc/html/qrqc.html].

61. Scythe - A Bayesian adapter trimmer. [https://github.com/vsbuffalo/ scythe].

62. Joshi N: Sickle - A windowed adaptive trimming tool for FASTQ files using quality. [https://github.com/najoshi/sickle].

63. Kent WJ: BLAT-the BLAST-like alignment tool. Genome Res 2002 12:656-664.

64. Li W, Godzik A: Cd-hit: a fast program for clustering and comparing large sets of protein or nucleotide sequences. Bioinformatics 2006, 22:1658-1659.

65. Altschul SF, Gish W, Miller W, Myers EW, Lipman DJ: Basic local alignment search tool. J Mol Biol 1990, 215:403-410.

66. BioPerl production scripts - Taxonomy. [http://www.bioperl.org/wiki/ Bioperl_scripts\#Taxonomy].

67. Eddy SR: Accelerated Profile HMM Searches. PLoS Comput Biol 2011, 7: e1002195.

68. Slater GSC: Exonerate software. [http://www.ebi.ac.uk/ guy/exonerate/].

69. Garrison E, Marth G: Haplotype-based variant detection from short-read sequencing. 2012 [http://arxiv.org/abs/1207.3907], arXiv.

70. Ellison CE, Hall C, Kowbel D, Welch J, Brem RB, Glass NL, Taylor JW: Population genomics and local adaptation in wild isolates of a model microbial eukaryote. Proc Natl Acad Sci USA 2011, 108:2831-2836.

71. Pages H, Aboyoun P, Gentleman R, DebRoy S: Biostrings: String objects representing biological sequences, and matching algorithms. $R$ package version 2241

72. Pages $H$, Aboyoun $P$, Lawrence M: IRanges: Infrastructure for manipulating intervals on sequences. $R$ package version 1144

73. USDA GrainGenes. [http://wheat.pw.usda.gov/GG2/WheatTranscriptome/].

doi:10.1186/gb-2013-14-6-r66

Cite this article as: Krasileva et al:: Separating homeologs by phasing in the tetraploid wheat transcriptome. Genome Biology 2013 14:R66.

\section{Submit your next manuscript to BioMed Central and take full advantage of:}

- Convenient online submission

- Thorough peer review

- No space constraints or color figure charges

- Immediate publication on acceptance

- Inclusion in PubMed, CAS, Scopus and Google Scholar

- Research which is freely available for redistribution

Submit your manuscript at www.biomedcentral.com/submit
Ciomed Central 